\title{
Fast Hybrid Method for the Study on Monostatic Scattering from Plasma-Coated Target above a Rough Surface
}

\author{
Rui Wang, Shui-Rong Chai, and Li-Xin Guo \\ School of Physics and Optoelectronic Engineering, Xidian University, Xian 710071, China \\ Correspondence should be addressed to Rui Wang; doufuruier@xidian.edu.cn
}

Received 11 February 2014; Accepted 11 April 2014; Published 8 May 2014

Academic Editor: Lorenzo Crocco

Copyright (C) 2014 Rui Wang et al. This is an open access article distributed under the Creative Commons Attribution License, which permits unrestricted use, distribution, and reproduction in any medium, provided the original work is properly cited.

\begin{abstract}
A fast hybrid method combining the reciprocity theorem with high frequency approximation algorithm is presented to deal with the problem of the monostatic scattering from a two-dimensional (2D) plasma-coated target above a one-dimensional (1D) Gaussian rough surface illuminated by the tapered incident wave. Without numerical solution of the polarization currents on the target and the surface, this hybrid method does not only save computer resources but also improve the computing speed significantly in contrast to the numerical methods. The hybrid method based on equivalent principle and reciprocity theorem, which is an improved and generalized version of the traditional multipath technique, can deal with the interactions between plasma-coated target and underlying surface much more accurately. Numerical results are given to verify the validity of the hybrid method, and then the hybrid method is employed to investigate the monostatic scattering from a plasma-coated airfoil above a Gaussian rough surface, including the effects of several key parameters on stealth performance, such as plasma angular frequency and electron collision frequency.
\end{abstract}

\section{Introduction}

With the rapid development of modern military electronic technology, the stealth performance becomes very important for the military target design. Existing target stealth technologies tend to make use of special aircraft shapes or of microwave-absorbing coatings, in order to reduce the radar cross-section (RCS) of the target. However, there are situations where these techniques may not be effective, such as the use of a wide range of frequencies in radar $[1,2]$. Plasma, which has a complex dielectric constant, can be used as a good absorber of electromagnetic (EM) waves over a wide range of frequencies and the plasma stealth has thus been used widely in military and space technology for its several advantages, exhibiting high attenuation over a large bandwidth and offering the possibility of being switched on and off at will by controlling the characteristic parameters of plasma properly.

In the past decades, study of EM waves scattering from target coated with plasma layers has received much attention, because of the complex influences of plasma on scattering response characteristics, which is of great potential in plasma stealth. Although the scattering from the plasma-coated target only is much needed and studied, the composite scattering from a plasma-coated target above a randomly rough surface is still important and should be carefully focused on because the radar echo information is mingled with much clutter of the complex environment, such as soil, sea surface, and even sands, which make the information extraction of target more difficult. For single rough surface scattering, analytical methods such as the Kirchhoff approximation (KA) [3] and small-perturbation method (SPM) [4] are often utilized. Considering the complicated interactions between the target and the rough surface, it is not easy to study the scattering of such composite model using these analytical approximation methods. A four-path model $[5,6]$ and modeexpansion method [7] have been employed by researchers to study the composite scattering. So many robust numerical methods and high frequency methods have been developed to deal with the problem of bistatic scattering from the composite model. Popular techniques such as method of moments (MoM) [8-10], finite element method (FEM) [11], and finite-difference time-domain (FDTD) [12] method are commonly used to study EM scattering from 2D target above 1D rough surface. To improve the efficiency of the classic 
approach, several integral equation-based techniques have been proposed, such as multiple sweep method of moments (MSMM) [13], multilevel UV method [14], fast multipole method (FMM) [15], and generalized forward-backward method (GFBM) [16], as well as its extensions GFBM/SAA [17] and GFBM/EPILE $[18,19]$. They all obtain the interactions between targets and rough surface by solving the Maxwell equations exactly. Besides pure numerical techniques, many studies have also contributed to the popularity and feasibility of hybrid analytical-numerical methods. In the territory of composite scattering from target and rough surface, MoM/SPM [20], KA-MoM [21], PO-MM [22], and EPILE-FBSA-PO2 [23] have been proposed to model the interactions between target and the underlying rough (sea) surface. It is worth mentioning that a recent published article [24] proposed another method to solve efficiently this kind of problem from PILE-PO based on MoM. It is interesting that, unlike [21, 22], PILE-PO can deal effectively with the fields scattered by a target below a rough surface due to the advantage of PILE method. PILE-PO is suitable for computing the field scattered by two targets, one of which is not directly illuminated. For instance, the scattering from a coated target, from a stack of two rough interfaces of infinite lengths separating homogeneous media (rough layer), or from a perfectly conducting target below a rough surface of infinite length and PILE-PO can be used to accelerate the calculation of the local interactions on the nonilluminated scatterer. These hybrid analytical-numerical methods based on MoM shown above can accelerate the computation greatly and hold certain accuracy under some conditions. Most of them only present examples of a perfectly conducting target above a rough (sea) surface. The hybrid FE-BI-KA method [25], which combines the KA with the hybrid finite elementboundary integral (FE-BI) method, is presented to consider bistatic scattering from a coated target above a rough sea surface. In this paper, a fast hybrid strategy combining the reciprocity theorem with high frequency approximation algorithm is presented to simulate the monostatic EM scattering from a plasma-coated target above a dielectric rough surface. This method, which makes it possible for physical optics (PO) to be used in composite scattering problems, improves the computing speed compared to the classical numerical methods, while retaining certain accuracy under some conditions. The bistatic scattering from a homogenous target over a rough surface has been studied frequently [20-25], whereas investigations on the monostatic scattering from a coated target over a dielectric rough surface have been paid little attention and not been fully discussed for the enormous computer resources and computation time. This is the main motive that drives the research on more powerful strategy.

The present work in this paper serves as a further extension of our previous study [25-28]. More attention has been given to dealing with the homogenous target in most of our previous studies except for [25]. The hybrid method presented in this paper could handle scattering problems involving an inhomogeneous target above a rough surface and, unlike [25], the main focus of our paper is on the study of the monostatic scattering, which has more practical significance than the bistatic scattering [25] in radar target detection.

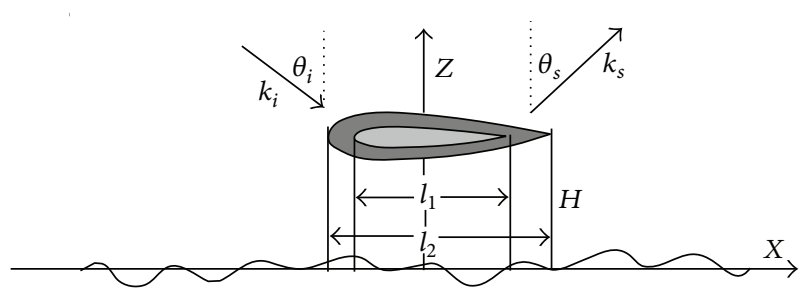

FIGURE 1: A plasma-coated airfoil over a rough surface.

Moreover, in this paper, a tapered incident plane wave is chosen instead of a plane wave to avoid artificial edge diffraction resulting from the finite length of the simulated rough surface and the multiple scattering up to 3 rd order was considered to make the results more believable.

This paper is organized as follows. The theoretical formulation of the hybrid method for calculating composite scattering from a plasma-coated target and rough surface is introduced in the second section. In the third section, the validation of hybrid method is verified and the numerical results of the composite model are discussed for several key parameters of plasma, such as electron collision frequency, plasma angular frequency, and the thickness of plasma layer. The fourth section ends with the conclusions of this paper and recommendations for further investigation in this topic.

\section{Scattering of Plasma-Coated Target above the Gaussian Rough Surface}

2.1. Multipath Scattering Model. Complex targets like ships and aircraft can be represented as collections of basic geometric elements, such as flat plates, cylinders, edges, or blended surfaces. Hence, it is reasonable to isolate the dominant sources of target echo and focus our attention upon individual elements instead of the composite target.

The basic geometry of EM scattering from a plasmacoated target above a $1 \mathrm{D}$ Gaussian rough surface is shown in Figure 1, where the plasma-coated target studied in this paper is a simplified scale model of an airfoil. The conducting airfoil has the cross-section $(y=0)$ of the NASA almond [29] with length $l_{1}=1 \mathrm{~m}$. A layer of plasma coating surrounds the airfoil within a boundary, which is also defined by the cross-section of NASA almond with length $l_{2}$. The airfoil structure is positioned at height $H$ above a truncated rough surface with the length of $L$. The rough surface profiles used in the study are realizations of a Gaussian random process and for simplicity are chosen to have an isotropic Gaussian correlation function. The resulting surface statistics are described completely by the surface (root-mean-square, rms) height $\delta$ and correlation length $l$.

Figure 2 illustrates the scattering mechanisms considered in the multipath model. Path (a) is a standard monostatic scattering path for the direct backscattered field from the rough interface, which is denoted by $\mathbf{E}_{1}$. Path (b) is for the direct backscattered field denoted by $\mathbf{E}_{2}$ from the target calculated as though it is located in free space. Path (c) is for the second order (2nd order) backscattered field, namely, 


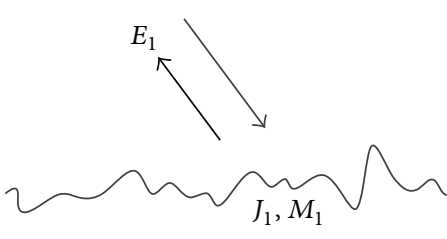

(a)

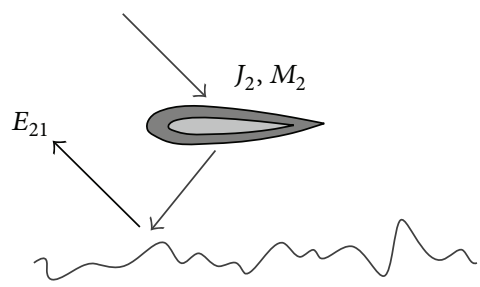

(d)

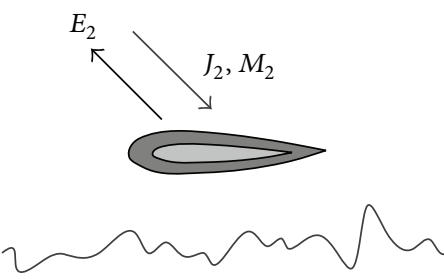

(b)

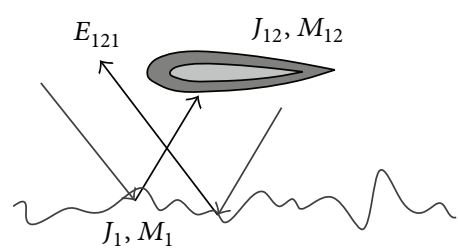

(e)

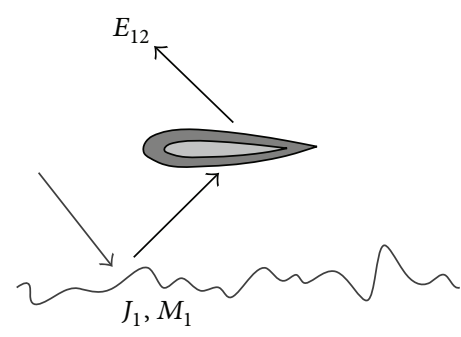

(c)

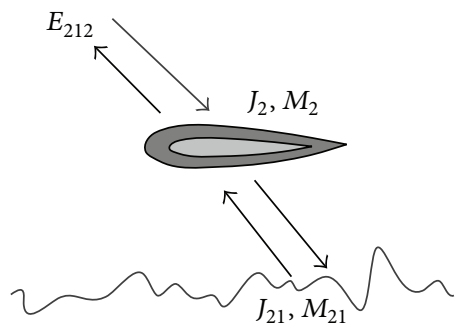

(f)

FIGURE 2: Scattering mechanic of multipath model.

"surface-target" backscattering, denoted by $\mathbf{E}_{12}$. Path (d) is also for the 2nd order backscattered field, namely, "targetsurface" backscattering, denoted by $\mathbf{E}_{21}$. Since Paths (c) and (d) are identical under time reversal, their contributions are equal. Both Path (e) and Path (f) are for the 3rd order backscattered field, namely, "surface-target-surface" and "target-surface-target" backscattering, denoted by $\mathbf{E}_{121}$ and $\mathbf{E}_{212}$, respectively. Note that Path (e) involves two reflections from the rough surface and a backscatter from the bottom surface of the target. However, Path (f) involves two diffractions from the target and a backscatter from the rough surface.

Then, the total backscattered field can be written as

$$
\mathbf{E}=\mathbf{E}_{1}+\mathbf{E}_{2}+\mathbf{E}_{12}+\mathbf{E}_{21}+\mathbf{E}_{121}+\mathbf{E}_{212} .
$$

2.2. Computation of the Primary Backscattered Field. In the paper, the problem is assumed to be $2 \mathrm{D}$ and the scalar equations may be used. However, it is convenient to deduce the vectorial formulations for $3 \mathrm{D}$ problem in the future, the equations in this paper are introduced the vectorial operators instead of scalar operators.

The time dependence is set to be $e^{-i \omega t}$ in our paper. Then the taper incident field applied can be written as

$$
\begin{aligned}
\mathbf{E}_{i}(\boldsymbol{\rho})=\widehat{y} \varphi_{i}= & \widehat{y} \exp \left\{i k\left(\widehat{k}_{i} \cdot \boldsymbol{\rho}\right)[1+w(\boldsymbol{\rho})]\right\} \\
& \times \exp \left\{-\left(x-z \tan \theta_{i}\right)^{2} / g^{2}\right\},
\end{aligned}
$$

where $w(\boldsymbol{\rho})=\left[2\left(x-z \tan \theta_{i}\right)^{2} / g^{2}-1\right] /\left(k_{i} g \cos \theta_{i}\right)^{2} ; g$ is the taper factor and $\theta_{i}$ denotes the incident angle. The incident magnetic field can be expressed as $\mathbf{H}_{i}(\boldsymbol{\rho})=\left[\nabla \times \mathbf{E}_{i}(\boldsymbol{\rho})\right] / i \omega \mu$. Assume the radius of curvature at each rough surface point is much larger than the wavelength of the incident wave, which means that the correlation length $l$ and the rms (root-meansquare) height $\delta$ of the rough surface conform to $k l>6, l^{2}>$
$2.76 \delta \lambda$, respectively. For horizontal polarization, using PO method, the surface equivalent electric and magnetic currents density $\mathbf{J}_{1}, \mathbf{M}_{1}$ induced by the incident wave in the absence of the target can be expressed as

$$
\begin{aligned}
& \mathbf{J}_{1}\left(\boldsymbol{\rho}_{s}\right) \\
& \quad= \begin{cases}\left(1-R_{H 1}^{i}\right) \widehat{n}_{s} \times \mathbf{H}_{i}\left(\boldsymbol{\rho}_{s}\right) & \text { illuminated }\left(\widehat{n}_{s} \cdot \widehat{k}_{i}<0\right) \\
0 & \text { unilluminated }\left(\widehat{n}_{s} \cdot \widehat{k}_{i}>0\right),\end{cases}
\end{aligned}
$$

$$
\begin{aligned}
& \mathbf{M}_{1}\left(\boldsymbol{\rho}_{s}\right) \\
& \quad= \begin{cases}\left(1+R_{H 1}^{i}\right) \mathbf{E}_{i}\left(\boldsymbol{\rho}_{s}\right) \times \widehat{n}_{s} & \text { illuminated }\left(\widehat{n}_{s} \cdot \widehat{k}_{i}<0\right) \\
0 & \text { unilluminated }\left(\widehat{n}_{s} \cdot \widehat{k}_{i}>0\right),\end{cases}
\end{aligned}
$$

where the lower indexes " $s$ " indicate these vectors are related to the rough surface. $\widehat{n}_{s}$ and $R_{H 1}^{i}$ are the unit normal vector and the Fresnel reflection coefficient of the rough interface, respectively. $\widehat{k}_{i}$ is the incident wave vector. It is noteworthy that the surface currents will vanish when some segments of the rough surface or the target are not illuminated by the incident wave. In Figure 3, it is given that the rough surface has been divided into $N$ segments. In segment $2, \widehat{n}_{s} \cdot \widehat{k}_{i}<0$ shows segment 2 can be illuminated by the incident wave and $\widehat{n}_{s} \cdot \widehat{k}_{i}>$ 0 in segment 3 indicates it could not be illuminated by the incident wave. In other words, segment 3 is one of the shadow zones and the corresponding surface currents density $\mathbf{J}_{1}, \mathbf{M}_{1}$ is zero. 


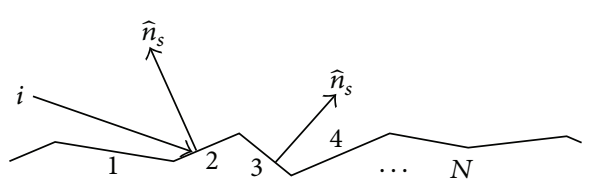

FIGURE 3: Distinguishing of the shadow zone on the rough surface.

Then $\mathbf{E}_{1}$, the backscattered field from the rough surface shown in Figure 2(a), can be given by Huygens' Principle as

$$
\begin{aligned}
\mathbf{E}_{1}\left(\boldsymbol{\rho}_{0}\right)= & \int_{s} \mathbf{M}_{1}\left(\boldsymbol{\rho}_{s}\right) \times \nabla G\left(\boldsymbol{\rho}_{0}, \boldsymbol{\rho}_{s}\right) d s \\
& +i \omega \mu\left(\overline{\bar{I}}+\frac{\nabla \nabla}{k^{2}}\right) \int_{s} G\left(\boldsymbol{\rho}_{0}, \boldsymbol{\rho}_{s}\right) \cdot \mathbf{J}_{1}\left(\boldsymbol{\rho}_{s}\right) d s .
\end{aligned}
$$

Here $\overline{\bar{I}}$ is the unit dyad. $G\left(\boldsymbol{\rho}, \boldsymbol{\rho}^{\prime}\right)=(i / 4) H_{0}^{(1)}\left(k\left|\boldsymbol{\rho}-\boldsymbol{\rho}^{\prime}\right|\right)$ is $2 \mathrm{D}$ Green's function and $H_{0}^{(1)}(\cdot)$ is the zeroth-order Hankel function of the first kind. Similarly, when the isolated target is illuminated by the incident wave, the backscattered electric field $\mathbf{E}_{2}$ shown in Figure 2(b) can be obtained as

$$
\begin{aligned}
\mathbf{E}_{2}\left(\boldsymbol{\rho}_{0}\right)= & \int_{c} \mathbf{M}_{2}\left(\boldsymbol{\rho}_{s}\right) \times \nabla G\left(\boldsymbol{\rho}_{0}, \boldsymbol{\rho}_{c}\right) d c \\
& +i \omega \mu\left(\overline{\bar{I}}+\frac{\nabla \nabla}{k^{2}}\right) \int_{c} G\left(\boldsymbol{\rho}_{0}, \boldsymbol{\rho}_{c}\right) \cdot \mathbf{J}_{2}\left(\boldsymbol{\rho}_{c}\right) d c,
\end{aligned}
$$

where

$$
\begin{aligned}
& \mathbf{J}_{2}\left(\boldsymbol{\rho}_{c}\right) \\
& \quad= \begin{cases}\left(1-R_{H 2}^{i}\right) \widehat{n}_{c} \times \mathbf{H}_{i}\left(\boldsymbol{\rho}_{c}\right) & \text { illuminated }\left(\widehat{n}_{c} \cdot \widehat{k}_{i}<0\right) \\
0 & \text { unilluminated }\left(\widehat{n}_{c} \cdot \widehat{k}_{i}>0\right),\end{cases}
\end{aligned}
$$

$$
\begin{aligned}
\mathbf{M}_{2} & \left(\boldsymbol{\rho}_{c}\right) \\
= & \begin{cases}\left(1+R_{H 2}^{i}\right) \mathbf{E}_{i}\left(\boldsymbol{\rho}_{c}\right) \times \widehat{n}_{c} & \text { illuminated }\left(\widehat{n}_{c} \cdot \widehat{k}_{i}<0\right) \\
0 & \text { unilluminated }\left(\widehat{n}_{c} \cdot \widehat{k}_{i}>0\right),\end{cases}
\end{aligned}
$$

where the lower indexes " $c$ " indicate these vectors are related to the plasma-coated target. $\widehat{n}_{c}$ is the unit normal vector of the target. In order to simplify this problem, we suppose the face of the plasma-coated airfoil illuminated by the incident wave as a plane face and the Fresnel reflection coefficient $R_{H 2}^{i}$ may be solved approximately by the closed-form formula proposed by Kong [30].

2.3. Computation of the Coupling Backscattered Field. The computation of the coupling scattered field between the plasma-coated target and the underlying rough surface is the greatest challenge in the problem. The rough surface is always approximate to a flat reflector with the Fresnel reflection coefficient taking into account the roughness of the rough surface for calculating the coupling scattered fields in the traditional four-path technique [5] which may cause inaccurate calculation results. In this paper, the reciprocity theorem is employed to evaluate the rescattered coupling interaction between the plasma-coated target and the rough surface. The formulations to calculate the coupling scattered fields for horizontal polarization are shown in the following. When the coated target is illuminated by line currents source $\mathbf{J}_{e}$ and $\mathbf{M}_{m}$ placed at the back-far-zone observation point $\boldsymbol{\rho}_{0}$, the corresponding scattered fields $\mathbf{E}_{J e 2}$ and $\mathbf{H}_{M m 2}$ on the rough surface can be calculated by PO combining with the Huygens' Principle. When the target is illuminated by $\mathbf{J}_{e}$ shown in Figure 4(a), the surface equivalent electric and magnetic currents can be written as $\mathbf{J}_{e 2}$ and $\mathbf{M}_{e 2}$ and the corresponding scattered fields of $\mathbf{J}_{e 2}$ and $\mathbf{M}_{e 2}$ can be denoted by $\mathbf{E}_{J e 2}=$ $\mathbf{E}_{J e 21}+\mathbf{E}_{M e 21}$. After a careful derivation, the scattered fields $\mathrm{E}_{J e 2}$ on the rough surface can be written as

$$
\mathbf{E}_{J e 2}\left(\boldsymbol{\rho}_{s}\right)=\mathbf{E}_{J e 21}\left(\boldsymbol{\rho}_{s}\right)+\mathbf{E}_{M e 21}\left(\boldsymbol{\rho}_{s}\right)=\widehat{\mathbf{y}} \frac{\omega \mu k}{16} \sqrt{\frac{2}{\pi k \rho_{0}}} e^{i k \rho_{0}} e^{-i \pi / 4} \int_{c}\left[\begin{array}{c}
\left(1-R_{H 2}^{e}\right) J_{c 0}^{(N)}\left(\boldsymbol{\rho}_{c}\right) H_{0}^{(1)}\left(k\left|\boldsymbol{\rho}_{s}-\boldsymbol{\rho}_{c}\right|\right) \\
-i\left(1+R_{H 2}^{e}\right) M_{c 0}^{(N)}\left(\boldsymbol{\rho}_{c}\right) H_{1}^{(1)}\left(k\left|\boldsymbol{\rho}_{s}-\boldsymbol{\rho}_{c}\right|\right)\left(\widehat{n}_{c} \cdot \widehat{\mathbf{R}}_{s c}\right)
\end{array}\right] d c ;
$$

here, $H_{1}{ }^{(1)}(\cdot)$ is the first-order Hankel function of the one kind. Notably, subscripts " $c$ " and " $s$ " denote target and rough surface, respectively:

$$
\begin{gathered}
J_{c 0}^{(N)}\left(\boldsymbol{\rho}_{c}\right)=e^{-i k \widehat{\mathbf{k}}_{s} \cdot \boldsymbol{\rho}_{c}}\left(\widehat{n}_{c} \cdot \widehat{\mathbf{k}}_{s}\right), \\
M_{c 0}^{(N)}\left(\boldsymbol{\rho}_{c}\right)=e^{-i k \widehat{\mathbf{k}}_{s} \cdot \boldsymbol{\rho}_{c}}, \\
\widehat{\mathbf{R}}_{s c}=\frac{\boldsymbol{\rho}_{s}-\boldsymbol{\rho}_{c}}{\left|\boldsymbol{\rho}_{s}-\boldsymbol{\rho}_{c}\right|}=\frac{\left(x_{s}-x_{c}\right) \widehat{x}+\left(z_{s}-z_{c}\right) \widehat{z}}{\sqrt{\left(x_{s}-x_{c}\right)^{2}+\left(z_{s}-z_{c}\right)^{2}}} .
\end{gathered}
$$

When the target is illuminated by $\mathbf{M}_{m}$ shown in Figure 4(b), the surface equivalent electric and magnetic currents can be written as $\mathbf{J}_{m 2}$ and $\mathbf{M}_{m 2}$, which are the corresponding scattered fields of $\mathbf{J}_{m 2}$ and $\mathbf{M}_{m 2}$ that can be denoted by $\mathbf{H}_{M m 2}\left(\boldsymbol{\rho}_{s}\right)=\mathbf{H}_{J m 21}\left(\boldsymbol{\rho}_{s}\right)+\mathbf{H}_{M m 21}\left(\boldsymbol{\rho}_{s}\right)$. After a careful derivation, the scattered fields $\mathbf{H}_{M m 2}\left(\boldsymbol{\rho}_{s}\right)$ on the rough surface can be written as

$$
\begin{aligned}
\mathbf{H}_{M m 2}\left(\boldsymbol{\rho}_{s}\right)= & \mathbf{H}_{J m 21}\left(\boldsymbol{\rho}_{s}\right)+\mathbf{H}_{M m 21}\left(\boldsymbol{\rho}_{s}\right) \\
= & \int_{c} \nabla G\left(\boldsymbol{\rho}_{s}, \boldsymbol{\rho}_{c}\right) \times \mathbf{J}_{m 2}\left(\boldsymbol{\rho}_{c}\right) d c+i \omega \varepsilon \\
& \times \int_{c}\left(\overline{\bar{I}}+\frac{\nabla \nabla}{k^{2}}\right) G\left(\boldsymbol{\rho}_{s}, \boldsymbol{\rho}_{c}\right) \cdot \mathbf{M}_{m 2}\left(\boldsymbol{\rho}_{c}\right) d c .
\end{aligned}
$$




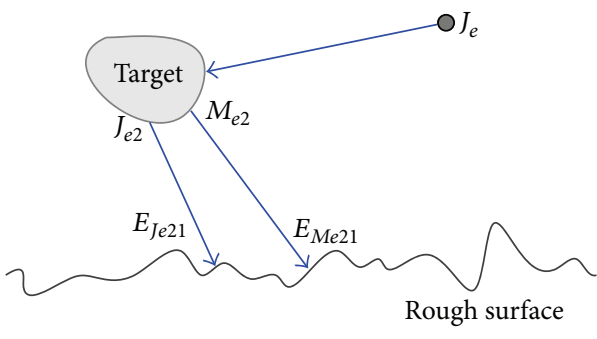

(a)

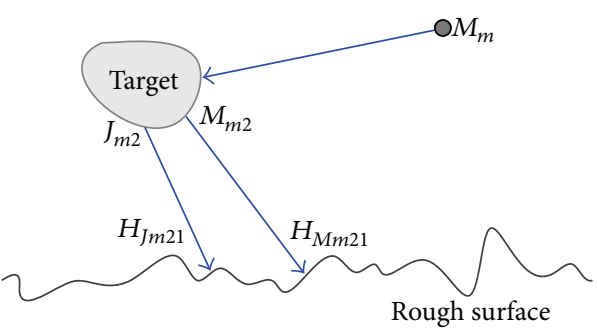

(b)

FIgURE 4: The scattered fields of the target illuminated by $\mathbf{J}_{e}$ and $\mathbf{M}_{m}$.

According to the reciprocity theorem $[26,30]$, we have

$$
\begin{gathered}
\widehat{\mathbf{y}} \cdot \mathbf{E}_{J 12}=\int_{s} \mathbf{J}_{1} \cdot \mathbf{E}_{J e 2} d s, \\
\widehat{\mathbf{q}} \cdot \mathbf{H}_{M 12}=\int_{s} \mathbf{M}_{1} \cdot \mathbf{H}_{M m 2} d s .
\end{gathered}
$$

Here, the unit horizontal polarization vector $\widehat{\mathbf{y}}$ and vertical polarization vector $\widehat{\mathbf{q}}$ are related by $\widehat{\mathbf{q}}=\widehat{\mathbf{k}}_{s} \times \widehat{\mathbf{y}}$; the electric current source $\mathbf{J}_{e}=\widehat{\mathbf{y}} \delta\left(\boldsymbol{\rho}-\boldsymbol{\rho}_{0}\right)$ and magnetic current source $\mathbf{M}_{m}=-\widehat{\mathbf{q}} \delta\left(\boldsymbol{\rho}-\boldsymbol{\rho}_{0}\right)$ were placed at the back-far-zone observation point $\rho_{0}$.

Then substituting (7) and (9) into (10) enables them to be written as

$$
\begin{aligned}
& \widehat{\mathbf{y}} \cdot \mathbf{E}_{J 12}=\frac{\omega \mu k}{16} \sqrt{\frac{2}{\pi k \rho_{0}}} e^{i k \rho_{0}} e^{-i \pi / 4} \int_{s}\left\{J_{1}\left(\boldsymbol{\rho}_{s}\right) \int_{c}\left[\begin{array}{c}
\left(1-R_{H 2}^{e}\right) J_{c 0}^{(N)}\left(\boldsymbol{\rho}_{c}\right) H_{0}^{(1)}\left(k\left|\boldsymbol{\rho}_{s}-\boldsymbol{\rho}_{c}\right|\right) \\
-i\left(1+R_{H 2}^{e}\right) M_{c 0}^{(N)}\left(\boldsymbol{\rho}_{c}\right) H_{1}^{(1)}\left(k\left|\boldsymbol{\rho}_{s}-\boldsymbol{\rho}_{c}\right|\right)\left(\widehat{\mathbf{n}}_{c} \cdot \widehat{\mathbf{R}}_{s c}\right)
\end{array}\right] d c\right\} d s \\
& \widehat{\mathbf{q}} \cdot \mathbf{H}_{M 12}=\int_{s} \mathbf{M}_{1} \cdot \mathbf{H}_{M m 2} d s \\
& =-\frac{k \omega \varepsilon}{16} \sqrt{\frac{2}{\pi k \rho_{0}}} e^{i k \rho_{0}} e^{-i \pi / 4}
\end{aligned}
$$

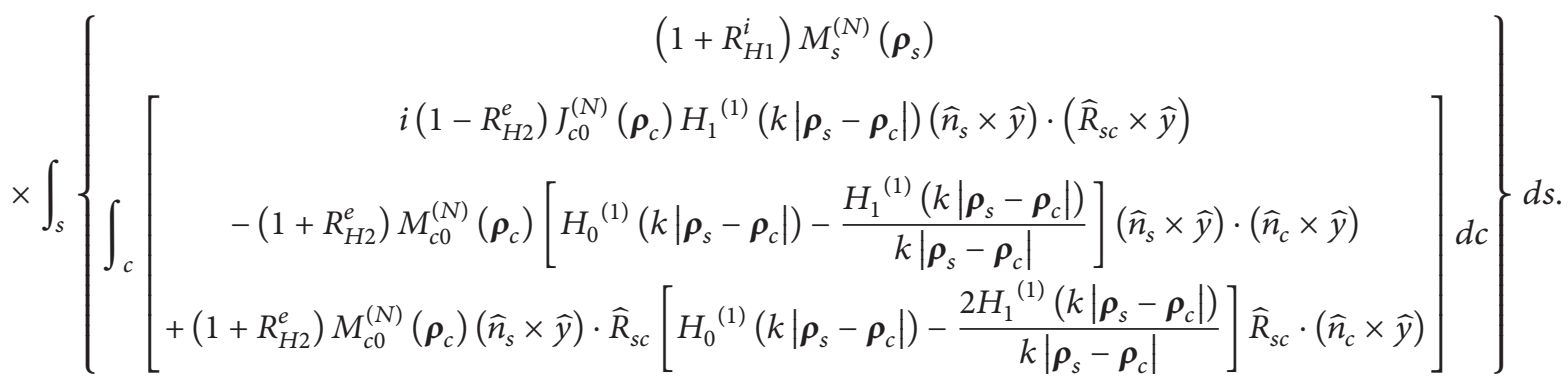

After obtaining $\mathbf{E}_{J 12}$ and $\mathbf{H}_{M 12}$, one of the 2nd orders monostatic scattering, the "surface-target" backscattered fields $\mathbf{E}_{12}=$ $\mathbf{E}_{J 12}+\mathbf{E}_{M 12}$ (shown in Figure 2(c)) can be obtained where the relation $\mathbf{E}_{M 12}=-\eta_{0} \widehat{\mathbf{k}}_{s} \times \mathbf{H}_{M 12}$ has been used. Here, $\eta_{0}$ is the wave impedance in the free space.

Similarly, when the underlying surface is illuminated by $\mathbf{J}_{e}, \mathbf{M}_{m}$, the corresponding scattered fields $\mathbf{E}_{J e 1}$ and $\mathbf{H}_{M m 1}$ on the rough surface can be also calculated by $\mathrm{PO}$ combined with the Huygens' Principle. When the target is illuminated by $\mathbf{J}_{e}$ shown in Figure 5(a), the surface equivalent electric and magnetic currents can be written as $\mathbf{J}_{e 1}$ and $\mathbf{M}_{e 1}$. The corresponding scattered fields of $\mathbf{J}_{e 1}$ and $\mathbf{M}_{e 1}$ can be denoted by $\mathbf{E}_{J e 1}=$ $\mathbf{E}_{J e 12}+\mathbf{E}_{M e 12}$. After a careful derivation, the scattered fields $\mathbf{E}_{J e 1}$ on the rough surface can be written as 


$$
\begin{aligned}
& \mathbf{E}_{J e 1}\left(\boldsymbol{\rho}_{c}\right)=\mathbf{E}_{J e 12}\left(\boldsymbol{\rho}_{c}\right)+\mathbf{E}_{M e 12}\left(\boldsymbol{\rho}_{c}\right)=\widehat{\mathbf{y}} \frac{\omega \mu k}{16} \sqrt{\frac{2}{\pi k \rho_{0}}} e^{i k \rho_{0}} e^{-i \pi / 4} \int_{s}\left[\begin{array}{c}
\left(1-R_{H 1}^{e}\right) J_{s 0}^{(N)}\left(\boldsymbol{\rho}_{s}\right) H_{0}^{(1)}\left(k\left|\boldsymbol{\rho}_{c}-\boldsymbol{\rho}_{s}\right|\right) \\
-i\left(1+R_{H 1}^{e}\right) M_{s 0}^{(N)}\left(\boldsymbol{\rho}_{s}\right) H_{1}^{(1)}\left(k\left|\boldsymbol{\rho}_{c}-\boldsymbol{\rho}_{s}\right|\right)\left(\widehat{n}_{s} \cdot \widehat{\mathbf{R}}_{c s}\right)
\end{array}\right] d s, \\
& J_{s 0}^{(N)}\left(\boldsymbol{\rho}_{s}\right)=e^{-i k \widehat{\mathbf{k}}_{s} \cdot \boldsymbol{\rho}_{s}}\left(\widehat{n}_{s} \cdot \widehat{\mathbf{k}}_{s}\right), \quad M_{s 0}^{(N)}\left(\boldsymbol{\rho}_{s}\right)=e^{-i k \widehat{\mathbf{k}}_{s} \cdot \boldsymbol{\rho}_{s}} \\
& \widehat{\mathbf{R}}_{c s}=\frac{\boldsymbol{\rho}_{c}-\boldsymbol{\rho}_{s}}{\left|\boldsymbol{\rho}_{c}-\boldsymbol{\rho}_{s}\right|}=\frac{\left(x_{c}-x_{s}\right) \hat{x}+\left(z_{c}-z_{s}\right) \widehat{z}}{\sqrt{\left(x_{c}-x_{s}\right)^{2}+\left(z_{c}-z_{s}\right)^{2}}} .
\end{aligned}
$$

When the target is illuminated by $\mathbf{M}_{m}$ shown in Figure 5(b), the surface equivalent electric and magnetic currents can be written as $\mathbf{J}_{m 1}$ and $\mathbf{M}_{m 1}$. The corresponding scattered fields of $\mathbf{J}_{m 1}$ and $\mathbf{M}_{m 1}$ can be denoted by $\mathbf{H}_{M m 1}\left(\boldsymbol{\rho}_{c}\right)=\mathbf{H}_{J m 12}\left(\boldsymbol{\rho}_{c}\right)+$ $\mathbf{H}_{M m 12}\left(\boldsymbol{\rho}_{c}\right)$. After a careful derivation, the scattered fields $\mathbf{H}_{M m 2}\left(\boldsymbol{\rho}_{s}\right)$ on the rough surface can be written as

$$
\begin{aligned}
\mathbf{H}_{M m 1}\left(\boldsymbol{\rho}_{c}\right)= & \mathbf{H}_{J m 12}\left(\boldsymbol{\rho}_{c}\right)+\mathbf{H}_{M m 12}\left(\boldsymbol{\rho}_{c}\right) \\
= & \int_{s} \nabla G\left(\boldsymbol{\rho}_{c}, \boldsymbol{\rho}_{s}\right) \times \mathbf{J}_{m 1}\left(\boldsymbol{\rho}_{s}\right) d s+i \omega \mathcal{\varepsilon} \\
& \times \int_{s}\left(\overline{\bar{I}}+\frac{\nabla \nabla}{k^{2}}\right) G\left(\boldsymbol{\rho}_{c}, \boldsymbol{\rho}_{s}\right) \cdot \mathbf{M}_{m 1}\left(\boldsymbol{\rho}_{s}\right) d s .
\end{aligned}
$$

Then substituting (12) and (14) into (15) enables these two equations above to be written as

According to the reciprocity theorem, we have

$$
\begin{gathered}
\widehat{\mathbf{y}} \cdot \mathbf{E}_{J 21}=\int_{c} \mathbf{J}_{2} \cdot \mathbf{E}_{J e 1} d s \\
\widehat{\mathbf{q}} \cdot \mathbf{H}_{M 21}=\int_{c} \mathbf{M}_{2} \cdot \mathbf{H}_{M m 1} d s .
\end{gathered}
$$

$$
\begin{aligned}
& \widehat{\mathbf{y}} \cdot \mathbf{E}_{J 21}=\frac{\omega \mu k}{16} \sqrt{\frac{2}{\pi k \rho_{0}}} e^{i k \rho_{0}} e^{-i \pi / 4} \int_{c}\left\{J_{2}\left(\boldsymbol{\rho}_{c}\right) \int_{s}\left[\begin{array}{c}
\left(1-R_{H 1}^{e}\right) J_{s 0}^{(N)}\left(\boldsymbol{\rho}_{s}\right) H_{0}^{(1)}\left(k\left|\boldsymbol{\rho}_{c}-\boldsymbol{\rho}_{s}\right|\right) \\
-i\left(1+R_{H 1}^{e}\right) M_{s 0}^{(N)}\left(\boldsymbol{\rho}_{s}\right) H_{1}^{(1)}\left(k\left|\boldsymbol{\rho}_{c}-\boldsymbol{\rho}_{s}\right|\right)\left(\widehat{\mathbf{n}}_{s} \cdot \widehat{\mathbf{R}}_{c s}\right)
\end{array}\right] d s\right\} d c \\
& \widehat{\mathbf{q}} \cdot \mathbf{H}_{M 21}=\int_{c} \mathbf{M}_{2} \cdot \mathbf{H}_{M m 1} d c \\
& =-\frac{k \omega \varepsilon}{16} \sqrt{\frac{2}{\pi k \rho_{0}}} e^{i k \rho_{0}} e^{-i \pi / 4}
\end{aligned}
$$

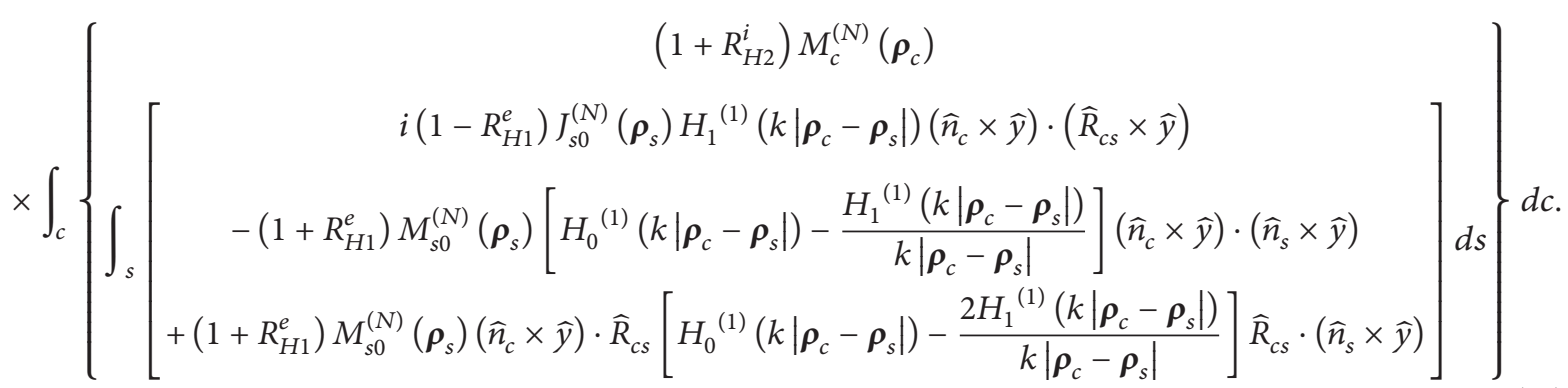

Then, the "target-surface" coupling backscattered fields $\mathbf{E}_{21}$ shown in Figure 2(d) can be obtained as $\mathbf{E}_{21}=\mathbf{E}_{J 21}+\mathbf{E}_{M 21}$ where the relation $\mathbf{E}_{M 21}=-\eta_{0} \widehat{\mathbf{k}}_{s} \times \mathbf{H}_{M 21}$ has been used.

Taking the scattered field of $\mathbf{E}_{i}$ from underlying rough surface as the incident field to illuminate the coated target, we can evaluate the equivalent surface electric and magnetic currents density $\mathbf{J}_{12}=\left(1-R_{H 2}^{1}\right) \widehat{n}_{c} \times \mathbf{H}_{1}\left(\boldsymbol{\rho}_{c}\right)$ and $\mathbf{M}_{12}=$
$\left(1+R_{H 2}^{1}\right) \mathbf{E}_{1}\left(\boldsymbol{\rho}_{c}\right) \times \widehat{n}_{c}$ on the target by PO. Based on the reciprocity theorem, we have

$$
\begin{gathered}
\widehat{\mathbf{y}} \cdot \mathbf{E}_{J 121}=\int_{c} \mathbf{J}_{12} \cdot \mathbf{E}_{J e 1} d c, \\
\widehat{\mathbf{q}} \cdot \mathbf{H}_{M 121}=\int_{c} \mathbf{M}_{12} \cdot \mathbf{H}_{M m 1} d c .
\end{gathered}
$$




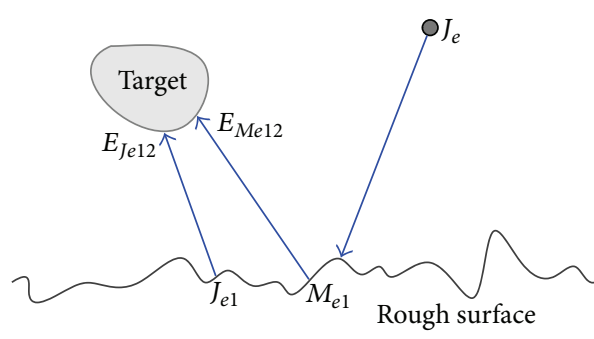

(a)

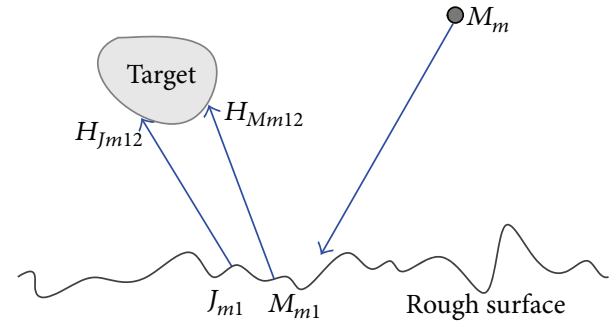

(b)

Figure 5: The scattered fields of the rough surface illuminated by $\mathbf{J}_{e}$ and $\mathbf{M}_{m}$.

By using this technique, one of the 3rd order monostatic scattering $\mathrm{E}_{121}$, named as "surface-target-surface" backscattered fields shown in Figure 2(e), can be given as $\mathbf{E}_{121}=$ $\mathbf{E}_{J 121}+\mathbf{E}_{M 121}$, where the relation $\mathbf{E}_{M 121}=-\eta_{0} \widehat{\mathbf{k}}_{s} \times \mathbf{H}_{M 121}$ has been used.

Similarly, taking the scattered field of $\mathbf{E}_{i}$ from the coated target as the incident field to illuminate the underlying rough surface, we can evaluate the equivalent surface electric and magnetic currents density $\mathbf{J}_{21}$ and $\mathbf{M}_{21}$ on the rough surface by PO. Based on the reciprocity theorem, we have

$$
\begin{gathered}
\widehat{\mathbf{y}} \cdot \mathbf{E}_{J 212}=\int_{s} \mathbf{J}_{21} \cdot \mathbf{E}_{J e 2} d s, \\
\widehat{\mathbf{q}} \cdot \mathbf{H}_{M 212}=\int_{s} \mathbf{M}_{21} \cdot \mathbf{H}_{M m 2} d s .
\end{gathered}
$$

So the "target-surface-target" coupling backscattered fields $\mathbf{E}_{212}$ shown in Figure 2(f)) can be gotten as $\mathbf{E}_{212}=$ $\mathbf{E}_{J 212}+\mathbf{E}_{M 212}$ where the relation $\mathbf{E}_{M 212}=-\eta_{0} \widehat{\mathbf{k}}_{s} \times \mathbf{H}_{M 212}$ has been used.

It is usual to pay more attention to the 2nd order scattering, which is always treated as the total coupling interactions in previous research. Since Paths (c) and (d) shown in Figure 2 are identical under time reversal, their contributions are equal, which indicates that the 2 nd order monostatic scattered field can be expressed as $2 \times \mathbf{E}_{12}$ or $2 \times \mathbf{E}_{21}$. However, it is found that Path (e) and (f) shown in Figure 2 are not identical, so it needs to calculate $\mathbf{E}_{121}$ and $\mathbf{E}_{212}$, respectively.

Using (4)-(5), (10), (15), and (17)-(20), the total scattered field of the composite model can be written as

$$
\begin{aligned}
\widehat{\mathbf{y}} \cdot \mathbf{E}= & \widehat{\mathbf{y}} \cdot \mathrm{E}_{1}+\widehat{\mathbf{y}} \cdot \mathrm{E}_{2}+\widehat{\mathbf{y}} \cdot \mathrm{E}_{21} \\
& +\widehat{\mathbf{y}} \cdot \mathrm{E}_{12}+\widehat{\mathbf{y}} \cdot \mathrm{E}_{121}+\widehat{\mathbf{y}} \cdot \mathrm{E}_{212} \\
= & \widehat{\mathbf{y}} \cdot \mathrm{E}_{1}+\widehat{\mathbf{y}} \cdot \mathrm{E}_{2}+2 \times \widehat{\mathbf{y}} \cdot \mathrm{E}_{21} \\
& +\widehat{\mathbf{y}} \cdot \mathrm{E}_{121}+\widehat{\mathbf{y}} \cdot \mathrm{E}_{212} .
\end{aligned}
$$

2.4. The Computation of the Monostatic Scattering Coefficient. The incident power $P_{i}$ received by the composite model is obtained by integrating over $x$ from $-\infty$ to $\infty$ :

$$
\begin{aligned}
P_{i} & =\int_{-\infty}^{\infty}\left[-\frac{1}{2 \eta_{0} k_{i}} \operatorname{Im}\left(E_{i} \frac{\partial E_{i}^{*}}{\partial z}\right)_{z=0}\right] d x \\
& =\frac{\cos \theta_{i}}{2 \eta_{0}} g \sqrt{\frac{\pi}{2}}\left[1-\frac{1+2 \tan ^{2} \theta_{i}}{2\left(k_{i} g \cos \theta_{i}\right)^{2}}\right] .
\end{aligned}
$$

On integration,

$$
P_{i}=\frac{\cos \theta_{i}}{2 \eta_{0}} g \sqrt{\frac{\pi}{2}}\left[1-\frac{1+2 \tan ^{2} \theta_{i}}{2\left(k_{i} g \cos \theta_{i}\right)^{2}}\right] .
$$

The power $P_{s}$ scattered by the composite model is obtained by

$$
P_{s}=\int_{-\pi / 2}^{\pi / 2} \rho_{0} \frac{-1}{2 \eta_{0} k_{i}} \operatorname{Im}\left\{E \nabla(E)^{*}\right\} d \theta_{s} .
$$

The monostatic scattering coefficient $\sigma\left(\theta_{s}\right)$ is defined so that

$$
\frac{P_{s}}{P_{i}}=\int_{-\pi / 2}^{\pi / 2} \sigma\left(\theta_{s}\right) d \theta_{s}=\frac{\int_{-\pi / 2}^{\pi / 2} \rho_{0}\left(-1 / 2 \eta_{0} k_{i}\right) \operatorname{Im}\left\{E \nabla(E)^{*}\right\} d \theta_{s}}{P_{i}}
$$

giving

$$
\sigma\left(\theta_{s}\right)=\frac{\rho_{0}\left(-1 / 2 \eta_{0} k_{i}\right) \operatorname{Im}\left\{E \nabla(E)^{*}\right\}}{P_{i}}
$$

With $P_{i}$ given by (22), we have

$$
\begin{aligned}
& \sigma\left(\theta_{s}\right) \\
& =\frac{-\rho_{0} \operatorname{Im}\left\{E \nabla(E)^{*}\right\}}{g k_{i} \cos \theta_{i} \sqrt{\pi / 2}\left[1-\left(1+2 \tan ^{2} \theta_{i}\right) /\left(2\left(k_{i} g \cos \theta_{i}\right)^{2}\right)\right]} .
\end{aligned}
$$

Putting (21) in (27), the monostatic scattering coefficient (MSC) of the composite model can be computed. 


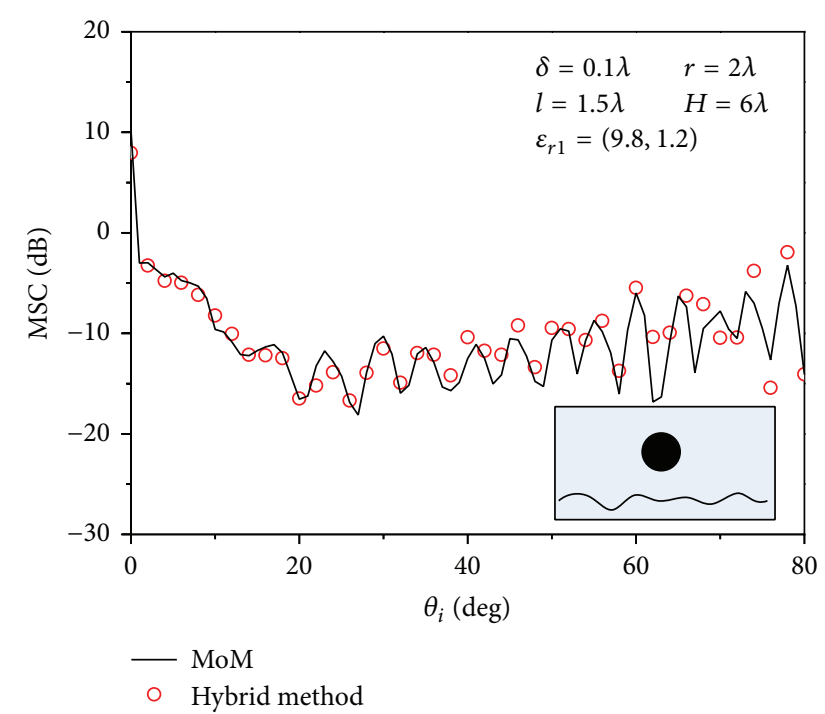

FIGURE 6: Monostatic scattering coefficient of a PEC cylinder above a dielectric rough surface.

\section{Method Verification}

In this section, we evaluate the accuracy of the proposed hybrid method by MoM and FDTD. Firstly, the hybrid method is verified by MoM when dealing with the problem of scattering from a PEC target above a dielectric surface. Figure 6 compares the MSC of a PEC cylinder and the underlying dielectric Gaussian rough surface calculated by using hybrid method with that by using MoM. Both the relevant parameters of the target and rough surface, such as radius and height of the cylinder ( $r$ and $H$ ), the surface dielectric permittivity $\left(\varepsilon_{r 1}\right)$, the rms $(\delta)$, and the correlation length $(l)$ of the rough surface, are measured in incident wavelength $\lambda$, which are all given in the textbox of the figure. The Gaussian rough surface is created by 20 Monte Carlo realizations in the following numerical simulations.

From the curves in Figure 6, it is obvious that the result obtained by hybrid method with the multiple scattering up to 3rd order shows acceptable agreement with that obtained by MoM for most incident angles except for big incident angles $\theta_{i}>70^{\circ}$. Indeed, the differences between the two curves in big incident angles are mainly due to two approximations used in our method. The first approximation is that the currents on each scatterer are computed by PO approximation. The multiple scattering and diffraction on the rough surface are ignored. Figure 7 gives the MSC of the rough surface alone by PO compared with that obtained by MoM, respectively. It is clear that there are big differences between PO and MoM when the incident angle $\theta_{i}>60^{\circ}$ because the multiple scattering is ignored by PO method. The MSC and BSC of the target alone by $\mathrm{PO}$ are compared with that by MoM in Figures 8 and 9. From the BSC curves in Figure 9 with the incident angle $\theta_{i}=90^{\circ}$, we can see that the results of the backscattering $\left(\theta_{s}=\right.$ $\left.90^{\circ}\right)$ and forescattering $\left(\theta_{s}=-90^{\circ}\right)$ by PO agree with that by MoM. Figure 8 verifies this point and it just shows the backscattering results by $\mathrm{PO}$ and MoM are identical. Notably,

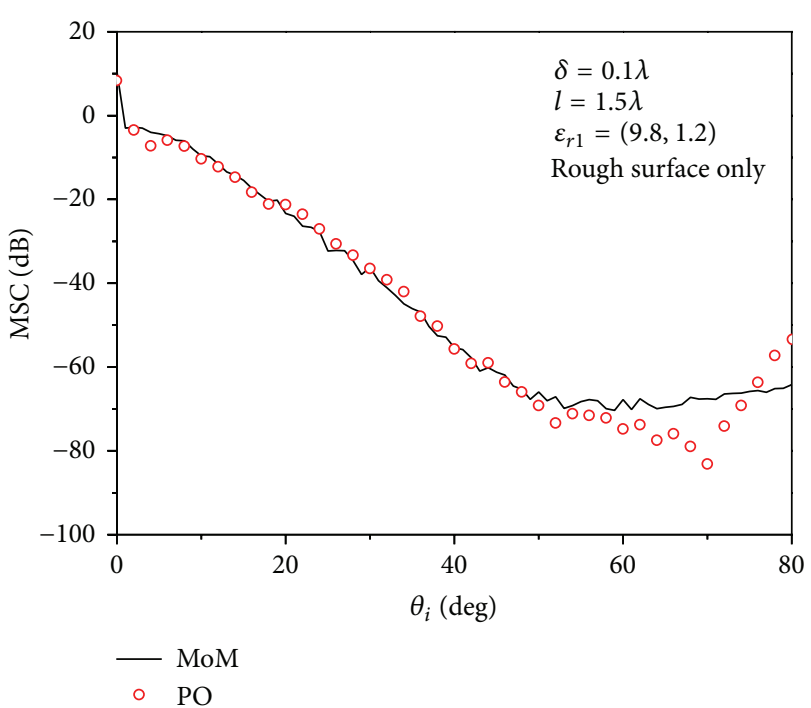

FIgURE 7: Monostatic scattering coefficient of a dielectric rough surface.

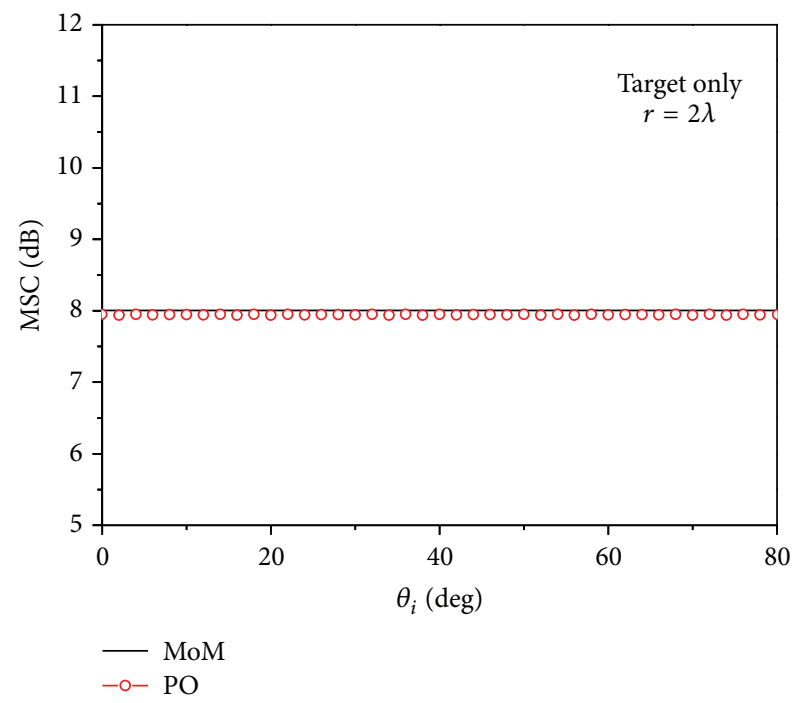

FIGURE 8: Monostatic scattering coefficient of a PEC cylinder.

the interaction between the target and the rough surface is closely related to the back- and forescattering of the target; thus acceptable results of the total MSC of the composite model can be gotten in Figure 6.

Moreover, the differences between the two curves in big incident angles are due to another approximation used in our method, which is that we considered the multiple interactions between the rough surface and the target only up to the $3 \mathrm{rd}$. This can be illustrated in Figure 10. In order to examine the validity of hybrid method when dealing with the problem of scattering from a coated target above a dielectric surface, as an example, we compute the bistatic scattering coefficient (BSC) of a dielectrically coated conducting cylinder above a Gaussian rough surface using the FDTD and hybrid method, respectively, in Figure 10. Relevant parameters, such as the 


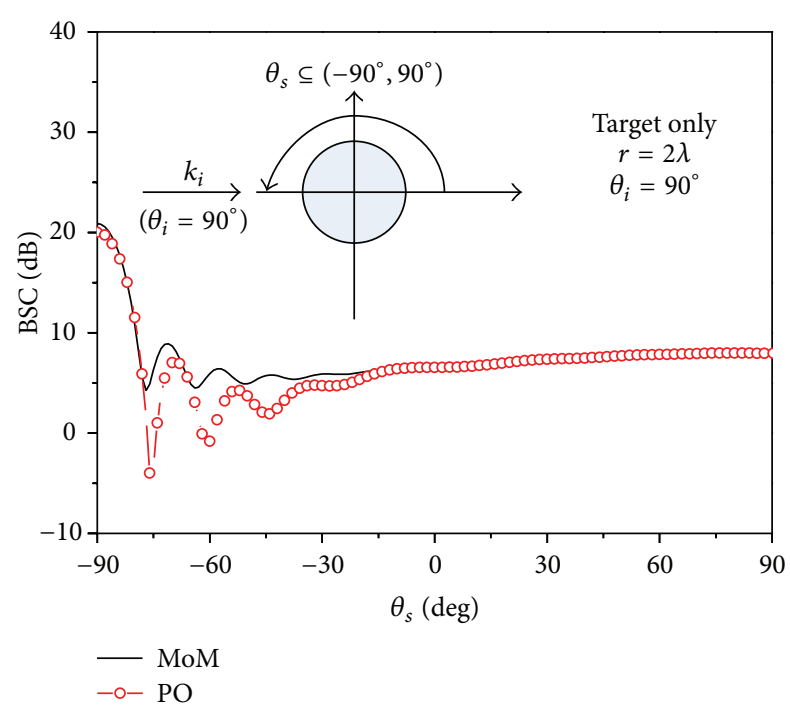

FIGURE 9: Bistatic scattering coefficient of a PEC cylinder.

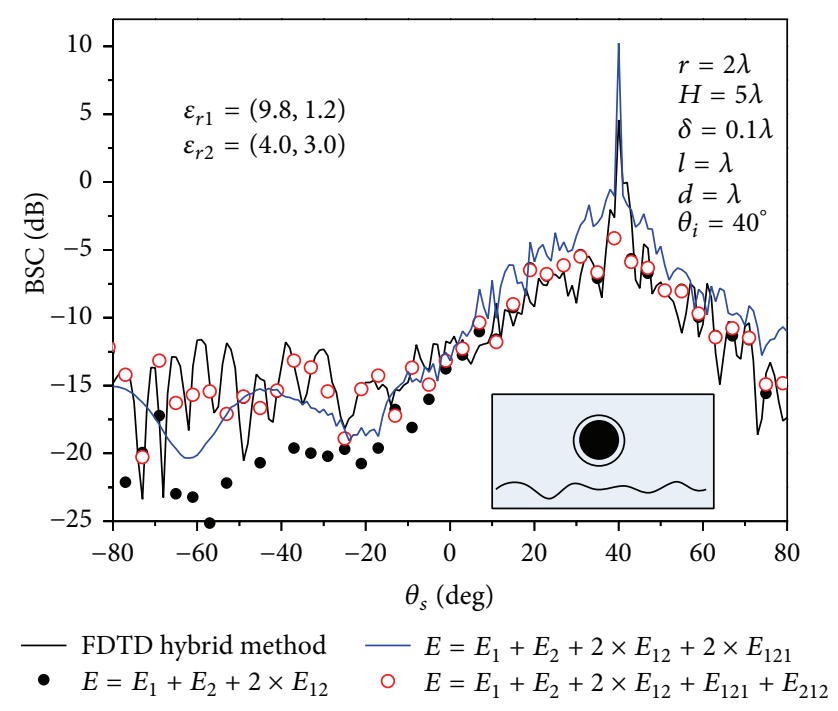

FIGURE 10: Bistatic scattering coefficient of a coated cylinder above a dielectric surface.

incident angle, the dielectric permittivity $\left(\varepsilon_{r 2}\right)$, and the thickness of the dielectrically layer $(d)$ coated on the target, are also given in the textbox of Figure 10 where the heavy black curve shows the result of FDTD. There are three curves of the results by hybrid method in the figure. The black filled circle represents the BSC calculated by hybrid method with the multiple interactions between both the scatterers which are accounted for up to the 2nd order; in other words, the total monostatic scattered field is expressed as $\mathbf{E}=\mathbf{E}_{1}+\mathbf{E}_{2}+2 \times \mathbf{E}_{12}$. It can be seen that this curve coincides with that of FDTD near the specular direction $\left(\theta_{i}=40^{\circ}\right)$ while it is not in satisfactory agreement with FDTD at most scattering angles especially near the monostatic scattering direction $\left(\theta_{s}=-40^{\circ}\right)$. Hence, the total monostatic scattering field with the multiple scattering up to 3rd order, which can be written as $\mathbf{E}=\mathbf{E}_{1}+$ $\mathbf{E}_{2}+2 \times \mathbf{E}_{12}+\mathbf{E}_{121}+\mathbf{E}_{212}$, is demonstrated by red circle in
Figure 10. It is observed that the BSC results with the multiple scattering up to 3rd order by hybrid method (red circle) are in more agreement with that by FDTD except for big scattered angle and the differences for big angles are mainly due to the fact that the multiple interactions between the target and the rough surface are only accounted for up to the 3rd order in our method.

Furthermore, the BSC result of the total monostatic scattering field $\mathbf{E}=\mathbf{E}_{1}+\mathbf{E}_{2}+2 \times \mathbf{E}_{12}+2 \times \mathbf{E}_{121}$ is also shown in Figure 10 which is depicted by thin blue curve. This curve indicates the 3 rd order scattering is expressed as $2 \times \mathbf{E}_{121}$ (or $2 \times \mathbf{E}_{212}$ ) instead of $\mathbf{E}_{121}+\mathbf{E}_{212}$. We can easily find that it rarely coincides with FDTD, which proves the fact mentioned in Section 2.3 that Paths (e) and (f) shown in Figure 2 are not identical and it needs to calculate $\mathbf{E}_{121}$ and $\mathbf{E}_{212}$, respectively.

Table 1 provides a comparison of the computational time between hybrid method and MoM for Figure 6, where the results for the larger scale rough surface case (with other parameters being the same) are also included. The simulations are performed on a PC platform with CPU of Intel I5 750, $4 \mathrm{~GB}$ memory, and Windows 7 64-bit operating system. From Table 1, it is found that our method shows a large substantial reduction of computational time. Moreover, it can be found that, with increasing the length of the rough surface, the hybrid method has great advantage in CPU time over MoM, which can also be seen from the ratio of the computational time by hybrid method and MoM in Table 1 .

Given that the 1D rough surface and the target are divided into $N$ and $M$ segments, respectively, a comparison of the complexity of these two methods is also shown in Table 1. The complexity of our method is mainly due to the solution of the 2nd and 3rd order backscattered field. In other words, the equations such as (10), (15), and (17)-(20) are closely related to the complexity. It is obvious that all of these equations involve double integral; thus the complexity is $O(N \times M)$. However, the complexity of MoM (using conjugate gradient method $(C G))$ is $O\left((2 N+M)^{2}\right)$.

\section{Numerical Results for a Plasma-Coated Airfoil above a Rough Surface}

This section studies the monostatic scattering characteristics of conducting but plasma-coated target above a rough surface with a Gaussian spectrum. The following results are based on an assumption that the frequency-dependent dielectric permittivity of the plasma coating layer is expressed by the single-pole Drude model, whereas the dielectric property of the lossy rough surface is independent of the frequencies in which we are interested. The Drude model can be given as

$$
\varepsilon_{r 2}(\omega)=1-\frac{\omega_{p}^{2}}{\omega^{2}+i \omega v},
$$

where $\omega_{p}$ is plasma angular frequency, which is determined by electron density and other parameters related to electron; $v$ is the electron collision frequency; and $\omega=2 \pi f$ is the angular frequency of the incidence wave. The relative dielectric permittivity of rough surface is assumed to be constant in all of the following simulations, which is $\varepsilon_{r 1}=9.6+i 1.2$. 
TABLE 1: Comparison of the computational time and complexity between the hybrid method and MoM.

\begin{tabular}{lccccc}
\hline $\begin{array}{l}\text { Length of the } \\
\text { rough surface }\end{array}$ & \multicolumn{2}{c}{ Time (seconds) } & \multicolumn{2}{c}{$\begin{array}{l}\text { Ratio of the computational time } \\
\text { by our method and MoM }\end{array}$} & $\begin{array}{c}\text { Complexity } \\
N \text { : rough surface; } M: \text { target }\end{array}$ \\
\hline $512 \lambda$ & 19 & 780 & $2.4 \%$ & Hybrid method & MoM $(\mathrm{CG})$ \\
$1024 \lambda$ & 61 & 3608 & $1.6 \%$ & $O(N \times M)$ & $O\left((2 N+M)^{2}\right)$ \\
\hline
\end{tabular}

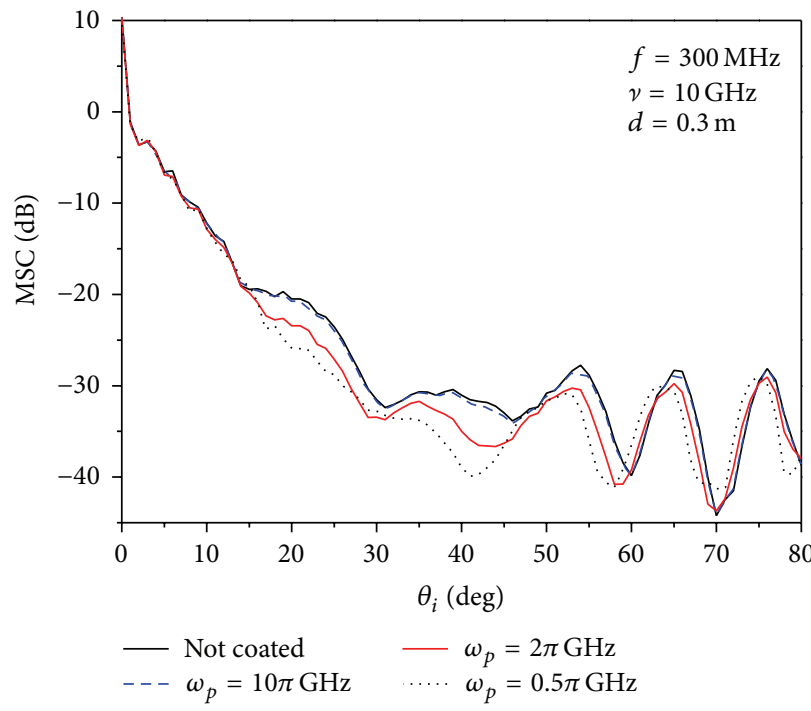

(a)

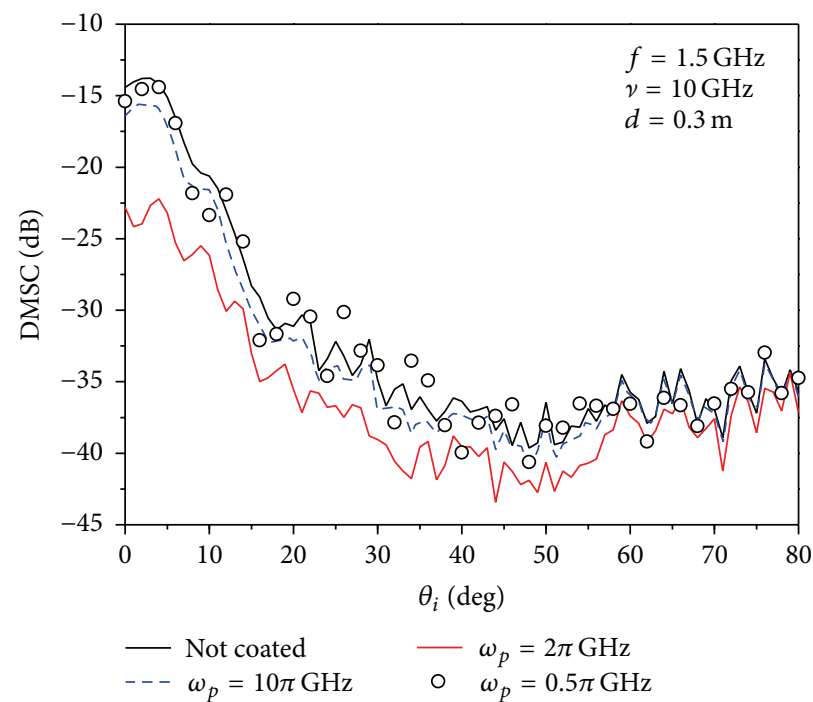

(c)

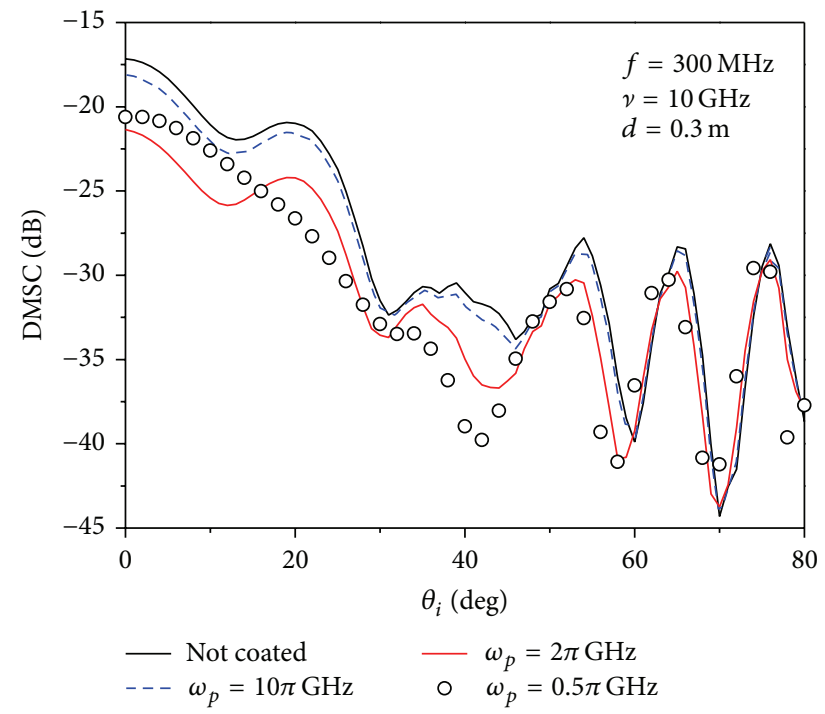

(b)

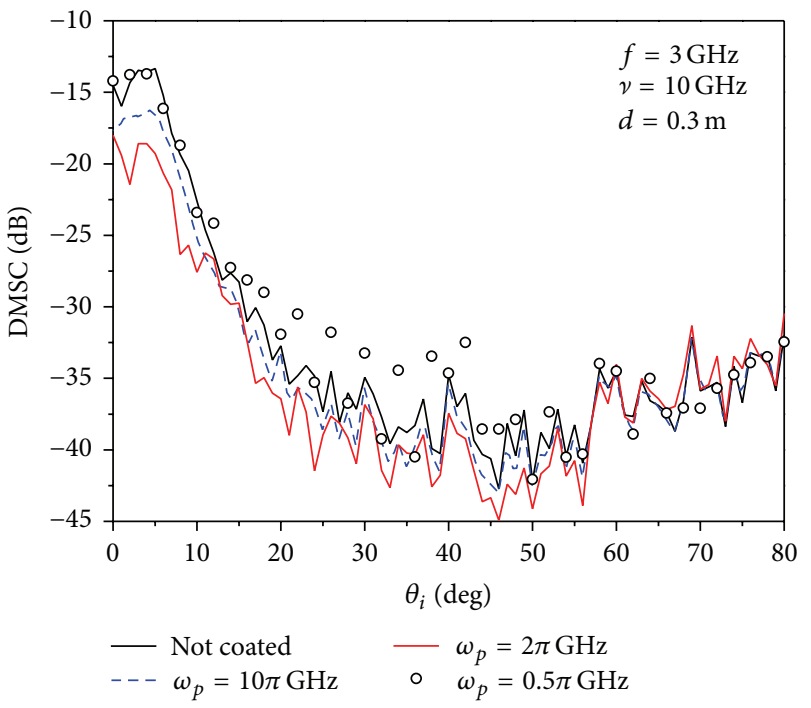

(d)

FIGURE 11: Monostatic scattering coefficient of plasma-coated airfoil above Gaussian rough surface: different plasma angular frequencies.

In the composite model, the airfoil is positioned at height $H=3 \mathrm{~m}$ above a truncated rough surface with the length of $L=375 \mathrm{~m}$. The tapered parameter $g=L / 4$. It is well known that the value of $g$ may become large when the incident angle increases [31], which is the most demanding conditions and can be shown as

$$
g=4\left[\frac{r}{\cos \theta_{i}}+H \operatorname{tg} \theta_{i}\right] .
$$

In other words, there may be different values of $g$ for different incident angles when solving the monostatic scattering from the composite model. In this paper the value of $g$ satisfies the requirement for $\theta_{i}=80^{\circ}$. Given $r=1 \mathrm{~m}, H=3 \mathrm{~m}$, and $\theta_{i}=$ $80^{\circ}$, the value of $g$ should be at least $91 \mathrm{~m}$. In fact, the length of the rough surface in our computation is $L=375 \mathrm{~m}$ and $g=L / 4=93.75 \mathrm{~m}>91 \mathrm{~m}$; (29) can be satisfied.

To consider the influence of plasma angular frequency $\omega_{p}$ on target stealth at three different incident frequencies: 


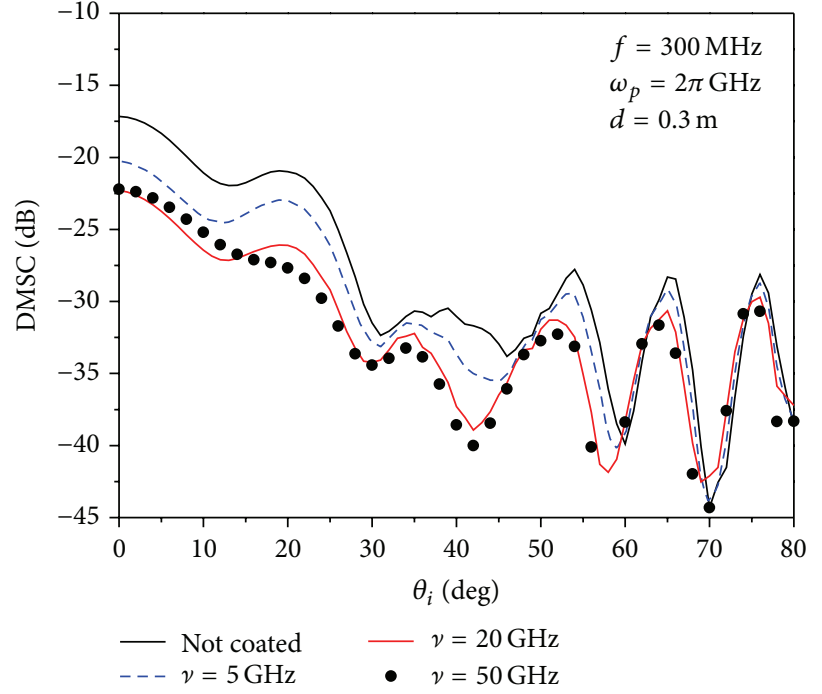

(a)

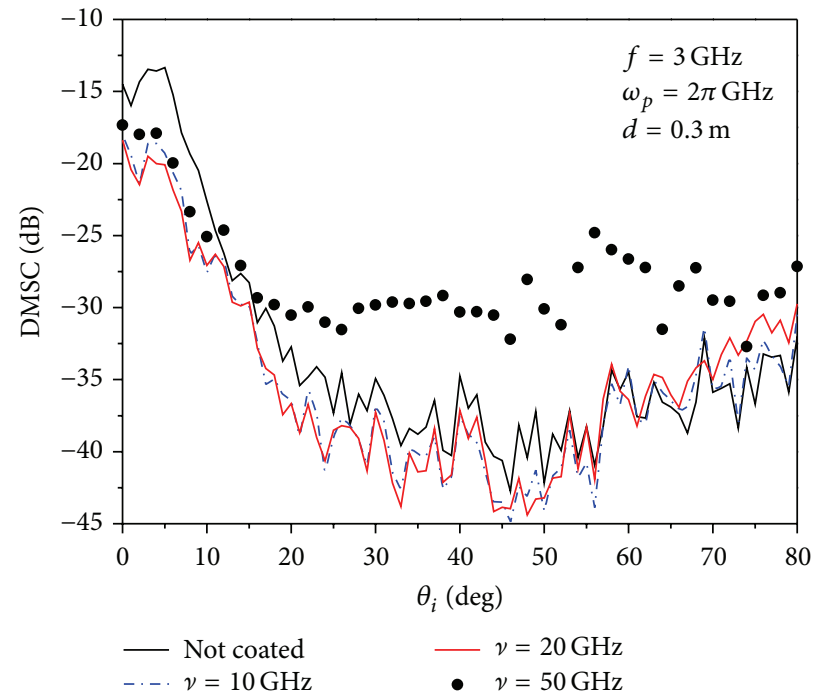

(b)

FIGURE 12: DMSC of the composite model for different electron collision frequency $v$.

$0.3 \mathrm{GHz}, 1.5 \mathrm{GHz}$, and $3 \mathrm{GHz}$, in Figure 6, simulations were carried out to compare the far-field reduction for different values of plasma angular frequency which ranges from $0.5 \pi \mathrm{GHz}$ to $10 \pi \mathrm{GHz}$ while electron collision frequency $v=$ $10 \mathrm{GHz}$. The thickness of the plasma layer is $d=0.3 \mathrm{~m}$.

Figure 11(a) shows the results of the MSC containing the total monostatic scattered field denoted by $\mathbf{E}=\mathbf{E}_{1}+\mathbf{E}_{2}+2 \times$ $\mathbf{E}_{12}+\mathbf{E}_{121}+\mathbf{E}_{212}$, whereas the results of the difference monostatic scattering coefficient (DMSC) containing the scattered field written as $\mathbf{E}_{2}+2 \times \mathbf{E}_{12}+\mathbf{E}_{121}+\mathbf{E}_{212}$ are plotted in Figures 11(b)-11(d). In Figure 11(a), one can observe that the MSC curves for different $\omega_{p}$ are reduced at the most incident angels except for the small incident angles, compared with the result without plasma coatings. However, the DMSC curves for different $\omega_{p}$ are reduced at all of the scattering angles especially at the small incident angles $\left(\theta_{i}<13^{\circ}\right)$ shown in Figure 11(b). The different performance of MSC and DMSC curves at the small incident angles results from the fact that the contribution of the direct scattered field $\mathbf{E}_{1}$ of the underlying rough surface to the total scattered field is great at the small incident angles; thus the MSC (containing $\mathbf{E}_{1}$ ) is not sensitive to the variation of the relevant parameters of the plasma coating on the target surface at the small incident angles. For this reason, in the following simulations, DMSC is used to show the influence of the plasma coating parameters on target stealth.

In Figure 11(b), the DMSC curves for $\omega_{p}=0.5 \pi \mathrm{GHz}$ and $\omega_{p}=2 \pi \mathrm{GHz}$ are below the other two curves which means better stealth effects at the incident frequency $f=500 \mathrm{MHz}$. To further consider the effects of plasma angular frequency at different incident frequencies, similar comparisons are shown in Figures 11(c) and 11(d) with $f=1.5 \mathrm{GHz}$ and $3 \mathrm{GHz}$, respectively. These simulations still choose plasma frequency from $0.5 \pi \mathrm{GHz}$ to $10 \pi \mathrm{GHz}$. It can be observed that the curves for $\omega_{p}=2 \pi \mathrm{GHz}$ are below the other curves which means better stealth effects. From the comparison of the curves for $\omega_{p}=0.5 \pi \mathrm{GHz}, \omega_{p}=2 \pi \mathrm{GHz}$, and $\omega_{p}=10 \pi \mathrm{GHz}$ in Figure 11, one can conclude that there is an optimum value of plasma frequency at given incidence and electron collision frequency and higher or lower plasma frequency than the optimum one (about $2 \pi \mathrm{GHz}$ in these examples) would lead to smaller MSC reduction.

In addition, a larger oscillation of the curves is observed at higher incident frequencies shown in Figures 11(c) and 11(d). This is mainly due to the significant effects of the local scattering which make the monostatic scattering field sensitive to the variation of incident angles.

The simulations in Figure 12 are focused on the effects of electron collision frequency $v$ upon the DMSC reduction due to plasma coatings. Plasma angular frequency is set as $\omega_{p}=$ $2 \pi \mathrm{GHz}$ and electron collision frequency is chosen from $v=$ $5 \mathrm{GHz}$ to $v=50 \mathrm{GHz}$. Similarly, increasing value of collision frequency would decrease DMSC at the lower incident frequency $f=500 \mathrm{MHz}$. However, the DMSC curve for $v=$ $50 \mathrm{GHz}$ at the higher incident frequency $f=3 \mathrm{GHz}$ is above the DMSC curve of the composite model without plasma coating. This means there is still an optimum value of collision frequency (about $20 \mathrm{GHz}$ in this example) at given incidence and plasma angular frequency.

To further explore the important scattering characteristics of this composite model, the dependence of DMSC on the thickness of the plasma coating $d$ is investigated in Figure 13. Plasma angular frequency and the electron collision frequency are set as $2 \pi \mathrm{GHz}$ and $10 \mathrm{GHz}$, respectively. The incident frequency is $f=500 \mathrm{MHz}$. It is clear that the amplitude of all these three DMSC curves decreases with the increase in $d$ and reaches the minimum values near $d=0.2 \mathrm{~m}$ which means the best stealth effect. The changes of the DMSC curves are not sensitive to the thickness of the plasma coating when $d>0.3 \mathrm{~m}$ which demonstrates that there is an optimum value of $d$. More simulations, whose results are not given here, were carried out and the authors found a similar conclusion for different incident angles. 


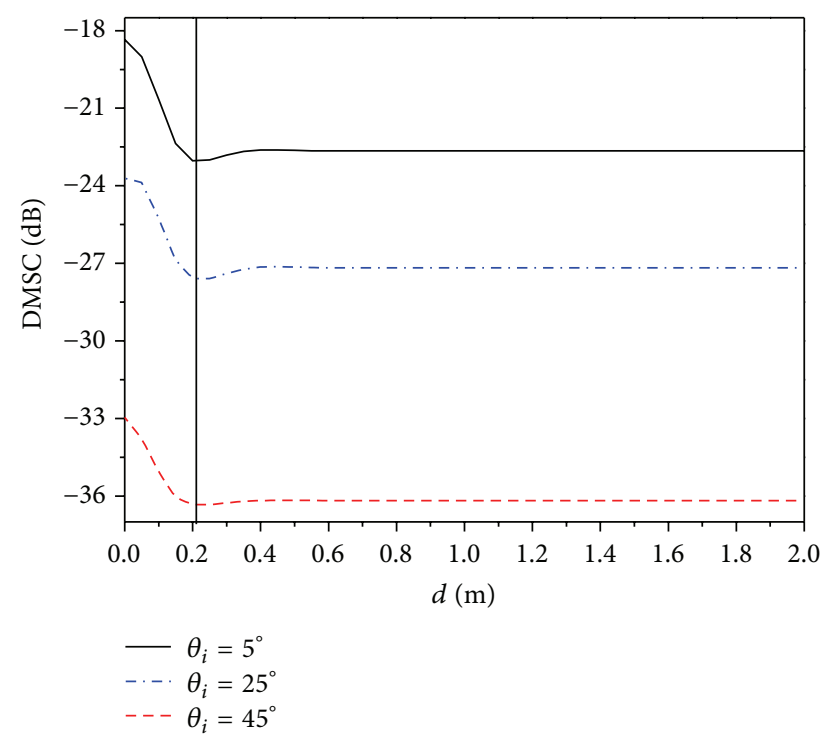

FIGURE 13: DMSC of the composite model for different thicknesses of plasma layer $d$.

\section{Conclusion}

The hybrid method can deal with the scattering from a plasma-coated target above a rough surface rapidly. In spite of the approximate nature of the high frequency approximation algorithm, this hybrid method can provide acceptable accuracy compared with classical numerical algorithm, such as MoM and FDTD. Simulations of the effects of a plasma layer on scattering pattern have been carried out by using this method, and several examples demonstrate the reduction of DMSC for coating by a plasma layer. Also, the influences of electron collision frequency, plasma angular frequency determined by electron density, and the thickness of the plasma layer on stealth performance have been considered. The optimum parameter of the plasma coatings of a given target at certain incidence wave frequency, which is of great essential in design and evaluation of protecting measures, could be found using a global searching method; however, choosing parameters of plasma coating which has good stealth performance for wide-band radar system and different environments is difficult. The method presented in this paper could be a starting point for such work. The future study could deal with the composite scattering from a 3D plasma-coated target above a $2 \mathrm{D}$ rough surface, which would be more interesting in practical applications.

\section{Conflict of Interests}

The authors declare that they have no financial or personal relationships with other people or organizations that can inappropriately influence their work; there are no professional or other personal interests of any nature or kind in any product, service, and/or company that could be construed as influencing the position presented in, or the review of, the paper.

\section{Acknowledgments}

This work was supported in part by the National Natural Science Foundation of China (Grant no. 61301070), the National Natural Science Foundation for Distinguished Young Scholars of China (Grant no. 61225002), the Specialized Research Fund for the Doctoral Program of Higher Education (Grant no. 20120203120023), and the Fundamental Research Funds for the Central Universities.

\section{References}

[1] E. F. Knott, J. F. Shaeffer, and M. T. Tuley, Radar Cross Section, Scitech, Raleigh, NC, USA, 2004.

[2] E. L. Mokole, M. Kragalott, and K. R. Gerlach, Ultra-Wideband Short-Pulse Electromagnetics 6, Kluwer Academic Publishers, Norwell, Mass, USA, 2003.

[3] H. Faure-Geors and D. Maystre, "Improvement of the Kirchhoff approximation for scattering from rough surfaces," Journal of the Optical Society of America A, vol. 6, no. 4, pp. 532-542, 1989.

[4] L.-X. Guo, Y. Liang, J. Li, and Z.-S. Wu, "A high order integral SPM for the conducting rough surface scattering with the tapered wave incidence-Te case," Progress in Electromagnetics Research, vol. 114, pp. 333-352, 2011.

[5] J. T. Johnson, "A numerical study of scattering from an object above a rough surface," IEEE Transactions on Antennas and Propagation, vol. 50, no. 10, pp. 1361-1367, 2002.

[6] E. A. Shtager, "An estimation of sea surface influence on radar reflectivity of ships," IEEE Transactions on Antennas and Propagation, vol. 47, no. 10, pp. 1623-1627, 1999.

[7] Y. Zhang, J. Lu, J. Pacheco Jr. et al., "Mode-expansion method for calculating electromagnetic waves scattered by objects on rough ocean surfaces," IEEE Transactions on Antennas and Propagation, vol. 53, no. 5, pp. 1631-1639, 2005.

[8] A.-Q. Wang, L.-X. Guo, and C. Chai, "Fast numerical method for electromagnetic scattering from an object above a largescale layered rough surface at large incident angle: vertical polarization," Applied Optics, vol. 50, no. 4, pp. 500-508, 2011.

[9] X. Wang and L.-W. Li, "Numerical characterization of bistatic scattering from PEC cylinder partially embedded in a dielectric rough surface interface: horizontal polarization," Progress in Electromagnetics Research, vol. 91, pp. 35-51, 2009.

[10] I. Simonsen, A. A. Maradudin, and T. A. Leskova, "Scattering of electromagnetic waves from two-dimensional randomly rough perfectly conducting surfaces: the full angular intensity distribution," Physical Review A, vol. 81, no. 1, Article ID 013806, 2010.

[11] P. Liu and Y.-Q. Jin, "An FEM approach with FFT accelerated iterative Robin boundary condition for electromagnetic scattering of a target with strong or weak coupled underlying randomly rough surface," IEEE Transactions on Antennas and Propagation, vol. 53, no. 12, pp. 4137-4144, 2005.

[12] L.-X. Guo, J. Li, and H. Zeng, "Bistatic scattering from a threedimensional object above a two-dimensional randomly rough surface modeled with the parallel FDTD approach," Journal of the Optical Society of America A, vol. 26, no. 11, pp. 2383-2392, 2009.

[13] D. Çolak and E. H. Newman, "The multiple sweep method of moments (MSMM) design of wide-band antennas," IEEE Transactions on Antennas and Propagation, vol. 46, no. 9, pp. 13651371, 1998. 
[14] P. Xu and L. Tsang, "Scattering by rough surface using a hybrid technique combining the multilevel UV method with the sparse matrix canonical grid method," Radio Science, vol. 40, no. 4, pp. 1-17, 2005.

[15] A.-Q. Wang, L.-X. Guo, and C. Chai, "Fast numerical method for electromagnetic scattering from an object above a largescale layered rough surface at large incident angle: vertical polarization," Applied Optics, vol. 50, no. 4, pp. 500-508, 2011.

[16] M. Rodriguez Pino, L. Landesa, J. L. Rodriguez, F. Obelleiro, and R. J. Burkholder, "The generalized forward-backward metod for analyzing the scattering from targets on ocean-like rough surfaces," IEEE Transactions on Antennas and Propagation, vol. 47, no. 6, pp. 961-969, 1999.

[17] Z.-X. Li, "Bistatic scattering from rough dielectric soil surface with a conducting object partially buried by using the GFBM/SAA method," IEEE Transactions on Antennas and Propagation, vol. 54, no. 7, pp. 2072-2080, 2006.

[18] G. Kubické, C. Bourlier, and J. Saillard, "Scattering by an object above a randomly rough surface from a fast numerical method: extended PILE method combined with FB-SA," Waves in Random and Complex Media, vol. 18, no. 3, pp. 495-519, 2008.

[19] Y. Liang, L. Guo, and Z. Wu, "The EPILE combined with the generalized-FBM for analyzing the scattering from targets above and on a rough surface," IEEE Antennas and Wireless Propagation Letters, vol. 9, pp. 809-813, 2010.

[20] Y. Zhang, Y. E. Yang, H. Braunisch, and J. A. Kong, "Electromagnetic wave interaction of conducting object with rough surface by hybrid SPM/MoM technique," Journal of Electromagnetic Waves and Applications, vol. 13, no. 7, pp. 983-984, 1999.

[21] H. Ye and Y.-Q. Jin, "A hybrid analytic-numerical algorithm of scattering from an object above a rough surface," IEEE Transactions on Geoscience and Remote Sensing, vol. 45, no. 5, pp. 11741179, 2007.

[22] S. Y. He and G. Q. Zhu, "A hybrid MM-PO method combining UV technique for scattering from two-dimensional target above a rough," Microwave and Optical Technology Letters, vol. 49, no. 12, pp. 2957-2960, 2007.

[23] G. Kubické and C. Bourlier, "A fast hybrid method for scattering from a large object with dihedral effects above a large rough surface," IEEE Transactions on Antennas and Propagation, vol. 59, no. 1, pp. 189-198, 2011.

[24] C. Bourlier, N. Pinel, and G. Kubické, "PILE method combined with $\mathrm{PO}$ for the scattering by coated cylinders, a rough layer and an object below a rough surface," Journal of Optical Society of America A, vol. 30, no. 9, pp. 1727-1737, 2013.

[25] J. Li, L.-X. Guo, Q. He, and B. Wei, "Investigation on scattering from a plasma-coated target over a rough sea surface using a multi-hybrid method," Waves in Random and Complex Media, vol. 22, no. 3, pp. 344-355, 2012.

[26] Y. Wang, L. Guo, and Z. Wu, "Electromagnetic scattering of gaussian beam by two-dimensional targets," Radio Science, vol. 42, no. 4, Article ID RS4012, 2007.

[27] R. Wang and L.-X. Guo, "Study on electromagnetic scattering from the time-varying lossy dielectric ocean and a moving conducting plate above it," Journal of the Optical Society of America $A$, vol. 26, no. 3, pp. 517-529, 2009.

[28] Y.-H. Wang, Y.-M. Zhang, M.-X. He, and L.-X. Guo, "Solution of scattering from rough surface with a $2 \mathrm{D}$ target above it by a hybrid method based on the reciprocity theorem and the forward-backward method," Chinese Physics B, vol. 17, no. 10, pp. 3696-3703, 2008.
[29] A. C. Woo, H. T. G. Wang, M. J. Schuh, and M. L. Sanders, "EM programmer's notebook-benchmark radar targets for the validation of computational electromagnetics programs," IEEE Antennas and Propagation Magazine, vol. 35, no. 1, pp. 84-89, 1993.

[30] J. A. Kong, Electromagnetic Wave Theory, Wiley-Interscience, 2002.

[31] H. Ye and Y.-Q. Jin, "Fast iterative approach to difference scattering from the target above a rough surface," IEEE Transactions on Geoscience and Remote Sensing, vol. 44, no. 1, pp. 108-115, 2006. 

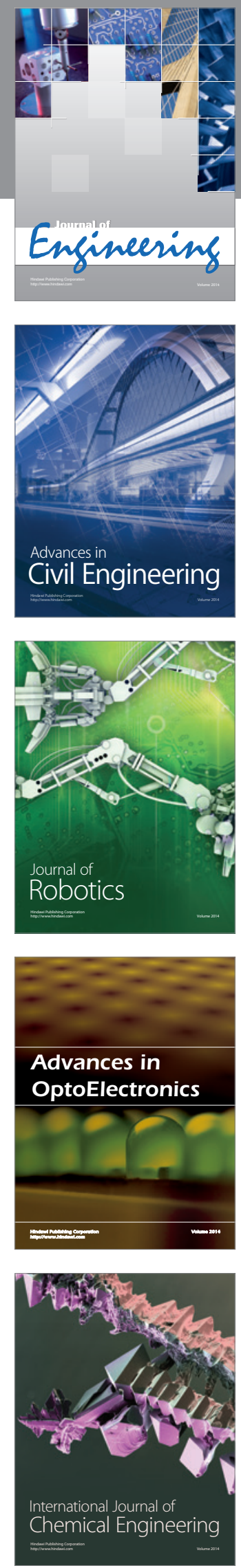

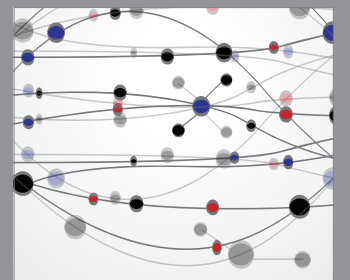

The Scientific World Journal
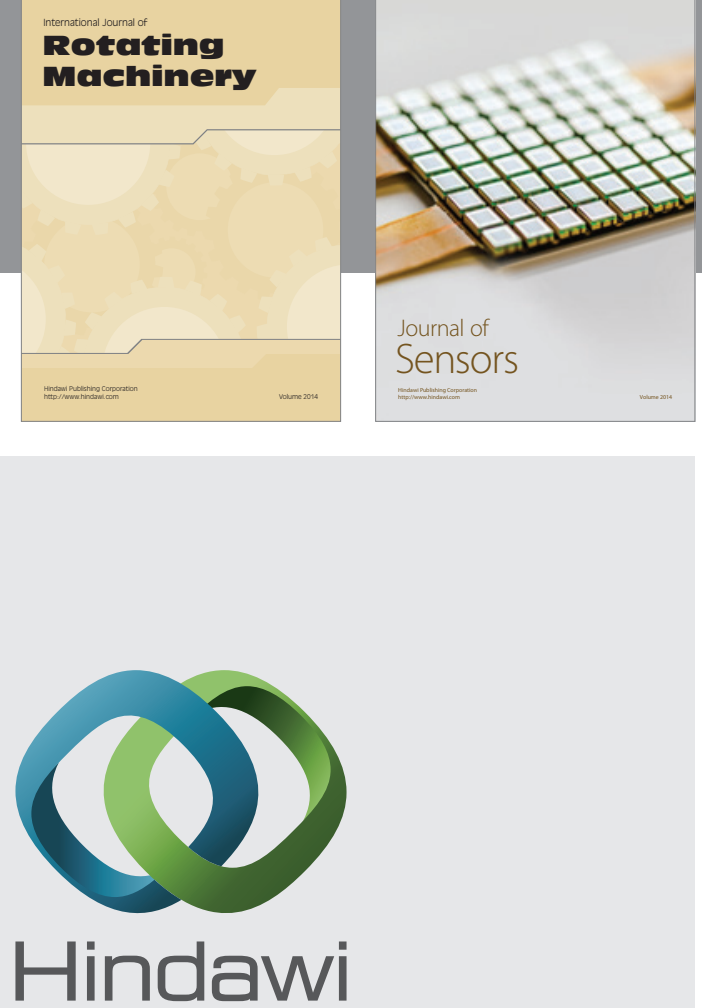

Submit your manuscripts at http://www.hindawi.com
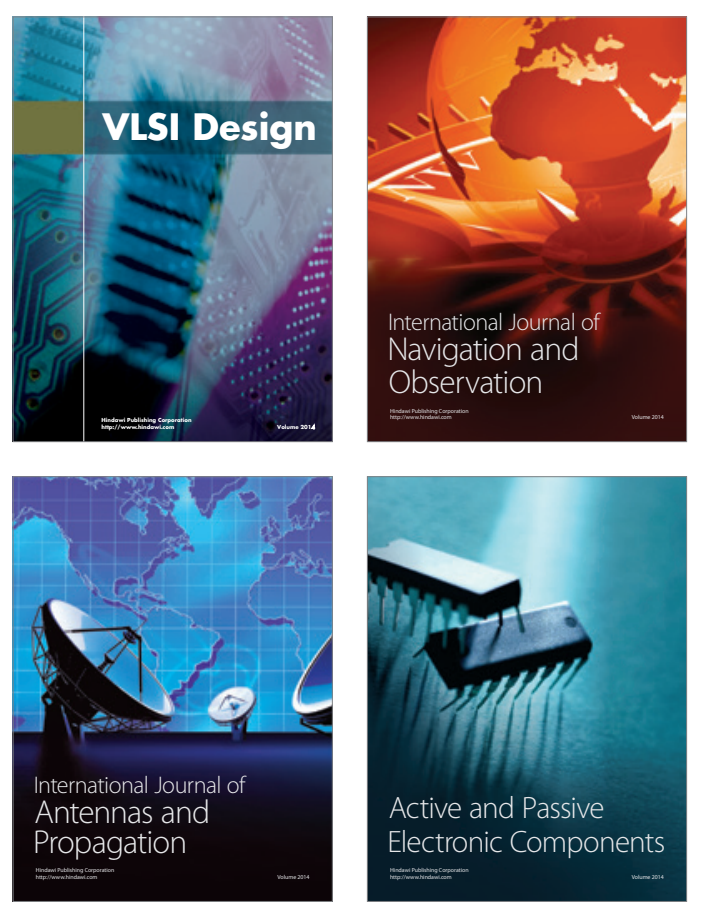
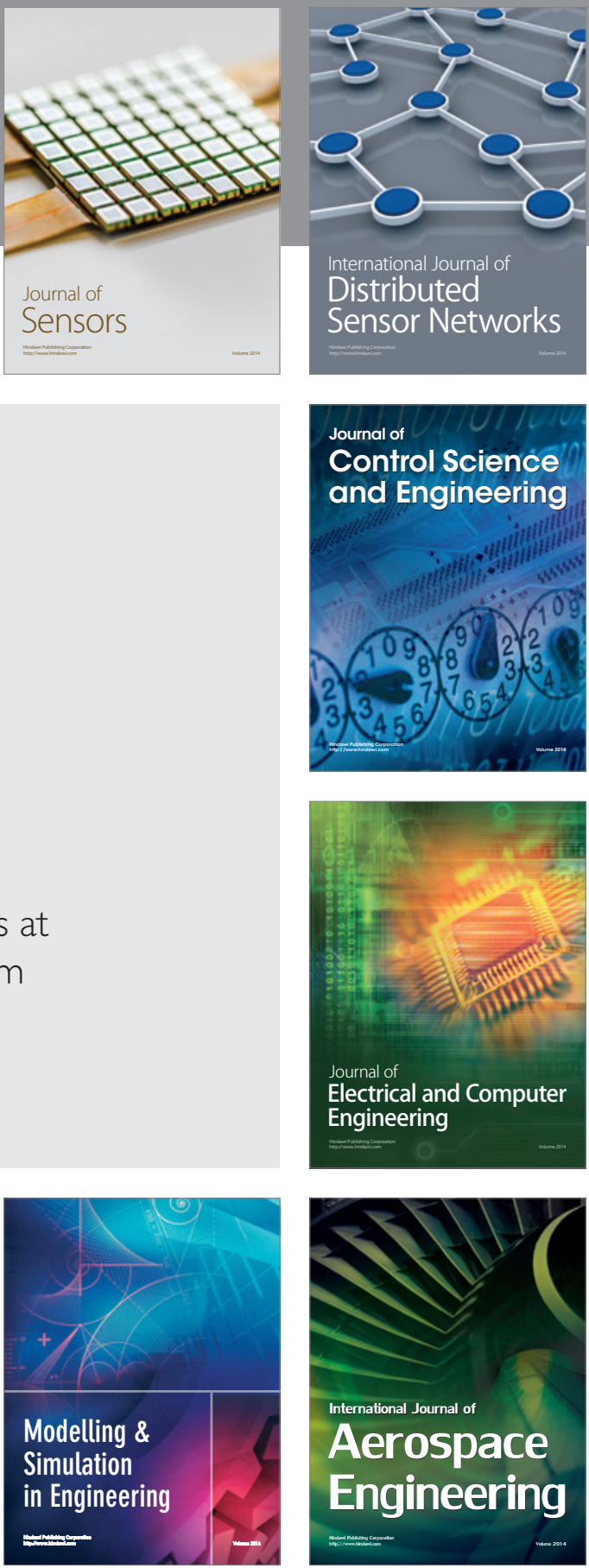

Journal of

Control Science

and Engineering
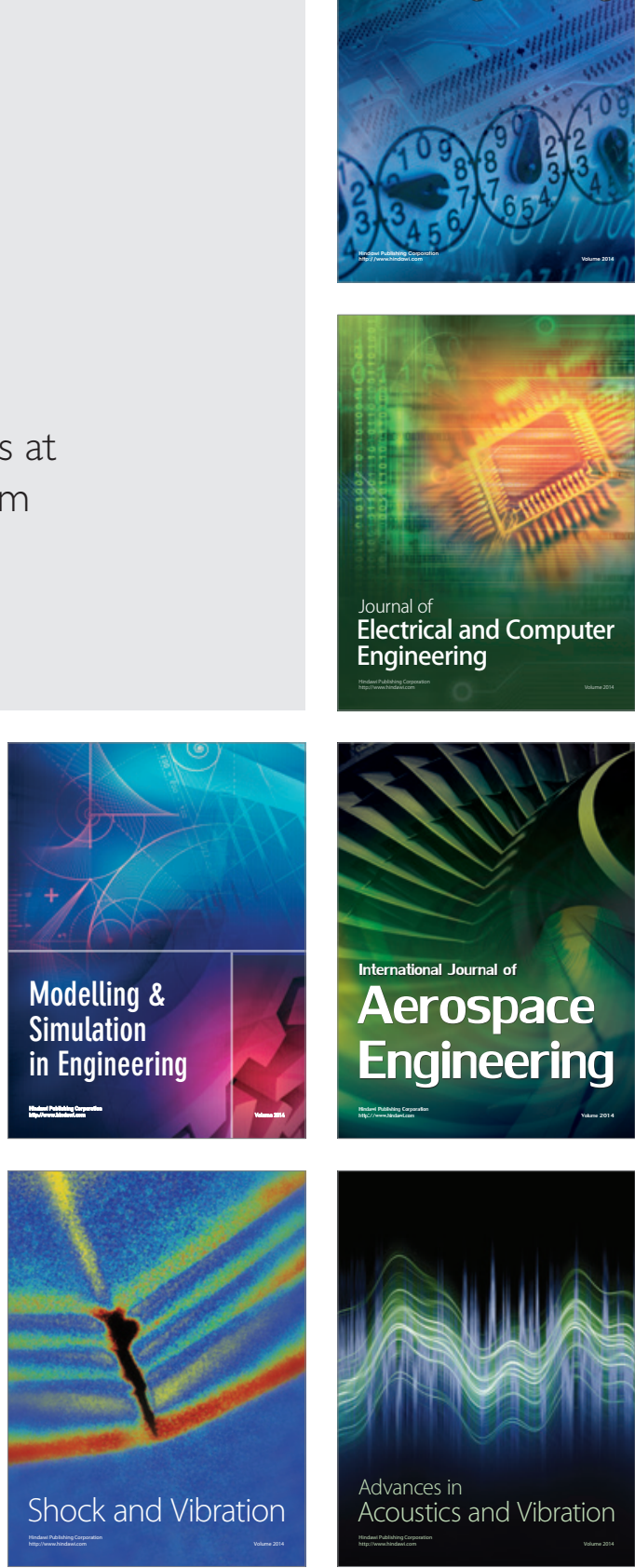